Meta

Journal des tradlucteurs

Translators' Journal

\title{
Théorie de langage, théorie de traduction
}

\section{Igor A. Mel'čuk}

Volume 23, numéro 4, décembre 1978

URI : https://id.erudit.org/iderudit/003621ar

DOI : https://doi.org/10.7202/003621ar

Aller au sommaire du numéro

Éditeur(s)

Les Presses de l'Université de Montréal

ISSN

0026-0452 (imprimé)

1492-1421 (numérique)

Découvrir la revue

Citer cet article

Mel'čuk, I. A. (1978). Théorie de langage, théorie de traduction. Meta, 23(4),

271-302. https://doi.org/10.7202/003621ar d'utilisation que vous pouvez consulter en ligne.

https://apropos.erudit.org/fr/usagers/politique-dutilisation/ 


\section{Théorie de langage, théorie de traduction*}

Considérons la thèse suivante qui, au premier abord, peut choquer mais qui, si on y réfléchit, s'avère plutôt banale ${ }^{1}$ :

(1) Tout usage du langage humain n'est qu'un procès constant de traduction; la langue (au sens saussurien) est une machine traductrice qui permet au sujet parlant d'exprimer ses pensées, désirs, impressions, etc. par la parole, et à l'auditeur, d'en extraire les pensées, désirs, impressions, etc. codés par l'émetteur.

Si on accepte la thèse (1) et surtout si on en tire toutes les conclusions intéressant la description linguistique ${ }_{2}$ on aboutira à des résultats qui ne sont plus banals ${ }^{2}$. En regardant l'émission des énoncés linguistiques, aussi bien que la perception de ceux-ci toujours comme une espèce de traduction, le linguiste se trouve en face de descriptions, ou de modèles, linguistiques, bien différents de ceux qu'on rencontre dans la linguistique soidisant traditionnelle et dans la linguistique dite moderne (grammaire transformationnelle, sémantique générative, etc.).

Je m'adresse, donc, aux linguistes ainsi qu'aux traducteurs pour les inciter à refaire de la linguistique une science de la traduction; je vais aussi proposer un modèle linguistique $\mathrm{t}_{1} / 2$ concret dont on pourrait se servir pour cette tâche.

La thèse (1) peut se réécrire sous la forme plus simple de l'équation suivante:

utilisation du langage $=$ traduction .

En formulant certaines généralités sur cette équation, puis, en en donnant une illustration, c'est-à-dire une description fragmentaire d'un modèle linguistique, pour le français, et, enfin, en passant en revue quelques difficultés de traduction universellement reconnues - afin de souligner leur pertinence primordiale pour la linguistique proprement dite, on va essayer de justifier la conclusion finale de l'article: LA LINGUISTIQUE EST LA SCIENCE DE TRADUCTION.

\section{LA LANGUE HUMAINE COMME SYSTĖME DE TRADUCTION}

Selon Roman Jakobson, «le sens d'un signe linguistique est sa traduction dans un autre signe » (Jakobson 1971b: 261). Ce n'est pas, d'ailleurs, l'unique passage chez Jakobson

* Cet article se base sur le texte d'une conférence prononcée dans la série des Confèrences Vanier à l'Université d'Ottawa (février 1978). 
où l'on trouve soulignée l'idée de traduction comme d'une opération linguistique, par excellence - ou, inversement, la conception de l'opération linguistique, aussi importante que la compréhension de la parole comme un acte de traduction. Et Jakobson n'est pas seul à insister depuis longtemps sur le fait que comprendre signifie traduire (fait presque évident mais malgré cela - ou, peut-être, à cause de cela - souvent négligé). Ce qu'il nous faut maintenant, c'est donner un sens plus précis à ces propos.

On posera comme point de départ le postulat (3):

(3) Dans chaque événement linguistique, (monologue, conversation, conférence, écriture d'un livre ou d'une lettre, lecture quelconque,... ) on doit distinguer deux aspects qui sont cruciaux pour le langage humain:

- un contenu, c'est-à-dire l'information à exprimer, qui passe (ou est supposée passer ) d'un interlocuteur à l'autre ;

- les phénomènes acoustiques ou graphiques qui portent ladite information.

On appellera les représentations symboliques des informations exprimées dans des énoncés linguistiques, les SENS, et les représentations symboliques des phénomènes physiques porteurs des sens, les TEXTES.

Remarque l. Les mots sens et texte seront donc dorénavant des termes techniques signifiant '( représentation de ) toute information exprimée dans des énoncés linguistiques ${ }_{1}{ }^{\prime}$ et '(représentation de) tout phénomène physique constituant l'aspect extérieur d'un énoncé linguistique ${ }_{1}^{\prime}$. Il ne faut pas leur chercher d'autres connotations, par exemple, évoquer l'opposition "sens vs signification » (d'après G. Frege), ou comprendre texte comme tout un discours cohérent opposé à phrase (dans le sens de la soi-disant text grammar), etc.

Remarque 2. Il semble superflu d'insister davantage sur la distinction substantielle entre les sens et les textes. Il suffit de se rappeler l'existence de la synonymie (un sens correspond à plusieurs textes) et de l'homonymie/polysémie (un texte correspond à plusieurs sens ) pour se convaincre de la nécessité de les séparer et de les étudier d'une façon indépendante.

S’appuyant sur (3), on va esquisser, sur le plan de langage, le tableau suivant.

Soit une communauté linguistique ${ }_{1}$. Il existe pour cette communauté trois objets abstraits :

un ensemble infini de sens, $\left\{\operatorname{SENS}_{\mathrm{i}} \mid 0 \leqslant \mathrm{i}<\infty\right\}$;

un ensemble infini de textes, $\left\{\operatorname{TEXTE}_{\mathrm{j}} \mid 0 \leqslant j<\infty\right\}$;

une correspondance multi-multivoque $\longleftrightarrow$ entre ces deux ensembles,

cette correspondance étant commune pour tous les membres de la communauté en question.

C'est précisément la correspondance $\Longleftrightarrow$ qu'on appelle, dans le langage courant, une LANGUE (française, anglaise, russe, esquimaude, ... ).

L'alinéa précédant peut donc s'écrire sous la forme de (4):

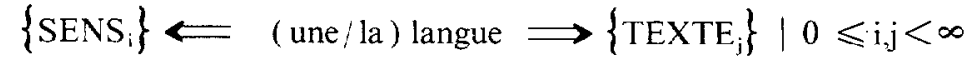


La tâche de la linguistique synchronique est, grossièrement, de décrire les langues différentes et de construire une théorie générale de la langue humaine. Il va sans dire que les descriptions et les théories linguistiques ${ }_{2}$ doivent être formelles, ce qu'on attend, d'ailleurs, de toute science.

Mais si on accepte (4), la description et la théorie des langues humaines deviennent la description et la théorie de CORRESPONDANCE entre les sens et les textes, ce qui veut dire que la description d'une langue (ou de la Langue, si l'on veut) ne peut être rien d'autre qu'un système de règles faisant correspondre à chaque sens donné tous les textes qui expriment, d'après les témoins de la langue, ce sens, et vice versa, à chaque texte donné tous les sens qui peuvent être exprimés par ce texte. La théorie des langues est alors une théorie de tels systèmes de règles.

Pourtant, un système de règles de correspondances constitue un système de traduction. En d'autres mots, une langue naturelle étant un système de correspondances entre les sens et les textes, utiliser une langue, c'est traduire les sens dans les textes, et les textes dans les sens. Par conséquent, décrire une langue, c'est décrire les règles de telles traductions, et la tâche de la linguistique synchronique et théorique est alors la construction et le perfectionnement des madèles linguistiques ${ }_{2}$ (= systèmes de règles) visant la traduction entre sens et textes: MODÈLES «SENS $\longleftrightarrow$ TEXTE» (MST).

Un MST pour une langue donnée est donc un système de règles formelles assurant la traduction de tout sens donné dans tous les textes correspondants et vice versa ${ }^{3}$. Comme on le voit,

un MST est un modèle TRANSLATIF qui s'oppose, précisément par ce trait, aux modèles GÉNÉRATIFS plus familiers de la linguistique moderne.

Pour établir des règles formelles de correspondances entre deux ensembles quelconques (les sens et les textes, dans notre cas), il faut que (les éléments de) ces ensembles soient décrits dans une langue formelle (ou dans des langues formelles).

Quant aux textes, ils ne posent aucun problème: il existe, depuis longtemps même, plusieurs langues formelles pour représenter les textes. Ce sont les écritures nationales et, plus récemment, les différentes transcriptions phonétiques et phonologiques, grâce auxquelles les textes peuvent s'écrire comme des suites de symboles empruntés à un alphabet.

Malheureusement, ce n'est pas le cas pour les sens: à l'heure actuelle, il n'y a pas de «transcription sémantique " semblable, mutatis mutandis, aux transcriptions phonétiques et phonologiques. Cela bloque le développement de la linguistique puisque, comme on vient de le voir, la linguistique synchronique a pour but de construire des MST, et un MST n'est pas même concevable sans une notation sémantique, une vraie langue formelle qui permette au chercheur de décrire les sens avec la même facilité et la même précision que celles dont il dispose pour le côté phonique du langage. Il s'ensuit que le premier pas du linguiste d'aujourd'hui doit consister à construire une LANGUE SÉMANTIQUE.

L'idée d'une langue sémantique n'est pas tout à fait nouvelle. Élaborée par Leibnitz sous la forme de Characteristica Universalis, elle réapparaît de nos jours dans l'œuvre de certains linguistes, dont on nommera, en premier lieu, A. Bogusławski et Anna Wierzbicka (yoir, par exemple, le livre Wierzbicka 1972 et les nombreux articles de cet auteur). Il faut 
avouer, cependant, que les langues sémantiques sont beaucoup plus populaires en dehors de la linguistique: ce sơnt surtout les informaticiens qui en construisent et s'en servent à leurs fins spécifiques, y compris la description des données des sciences concrètes (voir à ce propos les langues dites documentaires, telles que le SYNTOL de J. Gardin, et beaucoup d'autres). Or, c'est, à mon avis, la linguistique qui en aurait avant tout besoin. Je n'hésiterais même pas à affirmer que la construction d'une langue sémantique expérimentale pour la linguistique est à présent d'une priorité absolue. Les premières tentatives ont été déjà faites par les efforts conjoints de plusieurs savants parmi lesquels, à côté de Bogusławski et Wierzbicka déjà mentionnés, il faut citer A. Žolkovskij (1974: 155-234) et les autres auteurs de MPiPL 1964 (voir EoLS 1974): en effet, ce sont surtout leurs découvertes qui se trouvent à la base de la langue sémantique employée dans le MST concret que je vais décrire dans la présente communication.

Puisque, faute d'espace, il est impossible de parler de la langue sémantique (que je me propose d'utiliser) en détail, je me contenterai de n'en donner que quelques particularités déterminantes (et renvoyer le lecteur aux publications indiquées ci-dessus et dans la note 3 ):

1. Le formalisme de notre langue sémantique est le langage des graphes connexes orientés (au sens mathématique du terme), appelés rés e a ux: chaque expression dans cette langue, appelée représentation sémantique (SemR), contient un ensemble de points (= nœuds, ou sommets) liés par des flèches (= arcs).

2. Chaque nœud du graphe sémantique est étiqueté du nom d'une unité sémantique, ou sé $\mathrm{m}$ a $\mathrm{n}$ t è $\mathrm{me}$ (symboles entre guillemets ordinaires): c'est un sens élémentaire, sè me, ou bien un sens composé des sèmes ou d'autres sémantèmes. Un sémantème est une abréviation commode qui peut remplacer tout un sous-graphe sémantique; le plus souvent un sémantème constitue le signifié d'un lexème de la langue décrite ${ }^{4}$.

3. On distingue deux classes principales de sémantèmes: les fo n c t e u r s ( = prédicats, quantificateurs et connecteurs logiques) et les constantes (= noms propres et noms (de classes) d'objets).

4. Chaque arc du graphe sémantique est étiqueté d'un des nu méros dist in ct if s qui identifient les arguments des foncteurs correspondants: ' $X^{\prime} \bullet I^{\prime} P^{\prime} \stackrel{2}{\longrightarrow} \longrightarrow{ }^{\prime} Y^{\prime}$ signifie que les sémantèmes ' $\mathrm{X}$ ' et ' $\mathrm{Y}$ ' sont les $\mathrm{I}^{\mathrm{er}}$ et $2^{\mathrm{e}}$ arguments du sémantème ' $\mathrm{P}^{\prime}$, foncteur à deux places.

5. Le graphe ainsi étiqueté est appelé structure sémantique (SemS). À la SemS, on surimpose une structure sémantico-communicative (SemCommS): indication de thème $v s$ rhème (ce dont on parle dans l'énoncé représenté - ce qui est dit, ou communiqué, du thème); de mise en relief $v s$ absence de mise en relief (insistance particulière de la part du sujet parlant - absence de cette insistance), etc.

6. Les deux structures forment ensemble la représentation sémantique:

$$
\text { SemR }=\{\text { SemS, Sem-CommS }\} \text {. }
$$

Cette description de la langue sémantique choisie et de la SemR est fort sommaire et, en outre, nos recherches sont loin d'être terminées; par conséquent, on ne peut nullement insister sur les détails. Peut-être, il serait recommandable de mentionner deux points où les modifications semblent les plus imminentes: 
(i) Il faut probablement introduire, pour la SemR, les graphes d'ordre $n$ où $n>1$, c'est-à-dire, les graphes dont les sommets peuvent être aussi des graphes. Cette modification peut devenir nécessaire à propos des phénomènes comme le DOMAINE d'un quantificateur ou de la négation, etc.

(ii) Il faut probablement utiliser, dans notre SemR, de vraies variables et considérer toutes les constantes comme des foncteurs à une place; alors, par exemple, au lieu de 'bouteille' on écrirait toujours $\mathrm{X} .1$. bouteille' ('un X qui est une bouteille'). Cependant, pour la présente communication ces problèmes semblent être trop techniques.

Voilà un exemple de SemR:

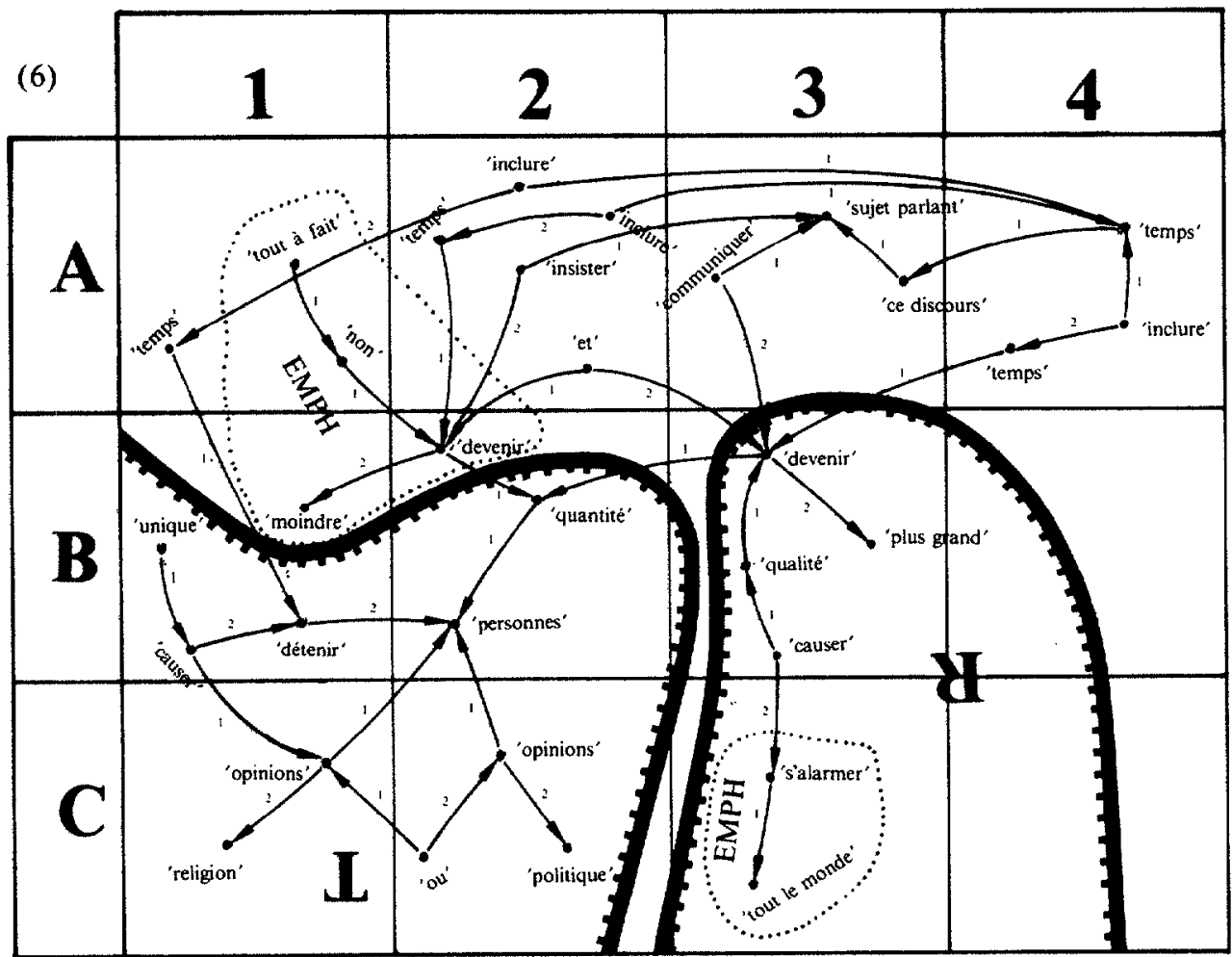

On peut verbaliser (6) de la façon suivantes:

(7) 'L'auteur de ce discours [voir A3: 'sujet parlant' 1 parle de [ $=$ thème, ou $\mathbf{I}$ ] la quantité des personnes qu'on détient au moment de ce discours [c'est-à-dire à présent: le temps [A 1] de la détention est inclus [A2] dans le temps [A4] de ce discours] à cause [BI] uniquement de leurs opinions sur la religion ou leurs opinions sur la politique [Cl-2];

L'auteur de ce discours insiste emphatiquement sur le fait que cette quantité ne devient pas tout à fait moindre [A 1-2, B 1-2] au moment du discours;

Il communique [A3] que [ = rhème, ou $\mathbf{~}$ ] cette quantité devient (toujours au moment du discours [B2, A3-4 ]) plus grande en mettant en relief le fait [EMPH dans C3.] que la qualité de cet accroissement cause que tout le monde s'alarme'. 
Comme une SemR a pour but de ne représenter que le SENS, ou le contenu, de l'énoncé donné, tout en ignorant sa forme, les moyens linguistiques,,$y$ compris le lexique, les exposants grammaticaux, l'ordre des éléments, les prosodies, etc. en sont éliminés comme tels et remplacés par des symboles homogènes: les unités sémantiques (= sémantèmes) et les unités communicatives (= thème vs rhème, mise en relief, etc.). Par exemple, le présent (une catégorie grammaticale du français) est rendu par 'le temps de la situation considérée est inclus dans le temps du discours', etc. ${ }^{6}$

Or, (7) est une lecture littérale de la SemR (6); quelles en seraient des francisations acceptables? Voilà quelques possibilités:

(8) Loin de diminuer, la détention de prisonniers d'opinion s'accroît de façon alurmante.

(9) Le nombre de personnes incarcérées uniquement pour leurs idées religieuses ou politiques, au lieu d'être à la baisse, augmente toujours tout en semant l'inquiétude (chez beaucoup de gens).

(10) Au lieu d'assister à une baisse du nombre de détenus d'opinion, on est témoin, tout ats contraire, d'une augmentation sensible de ce nombre, ce qui ne peut manquer de troubler l'opinion publique.

\begin{tabular}{|l|l|l|}
\hline Loin de & diminuer & détention \\
Au lieu de & être à la baisse & nombre de \\
Au contraire & perdre de l'importance & quantité de \\
$\mathrm{Ne} .$. nullement & perdre du terrain & \\
$\mathrm{Ne} . .$. aucunement & devenir moindre & \\
Pas du tout & devenir moins important & \\
& $\mathbf{6}$ & tomber \\
\hline
\end{tabular}

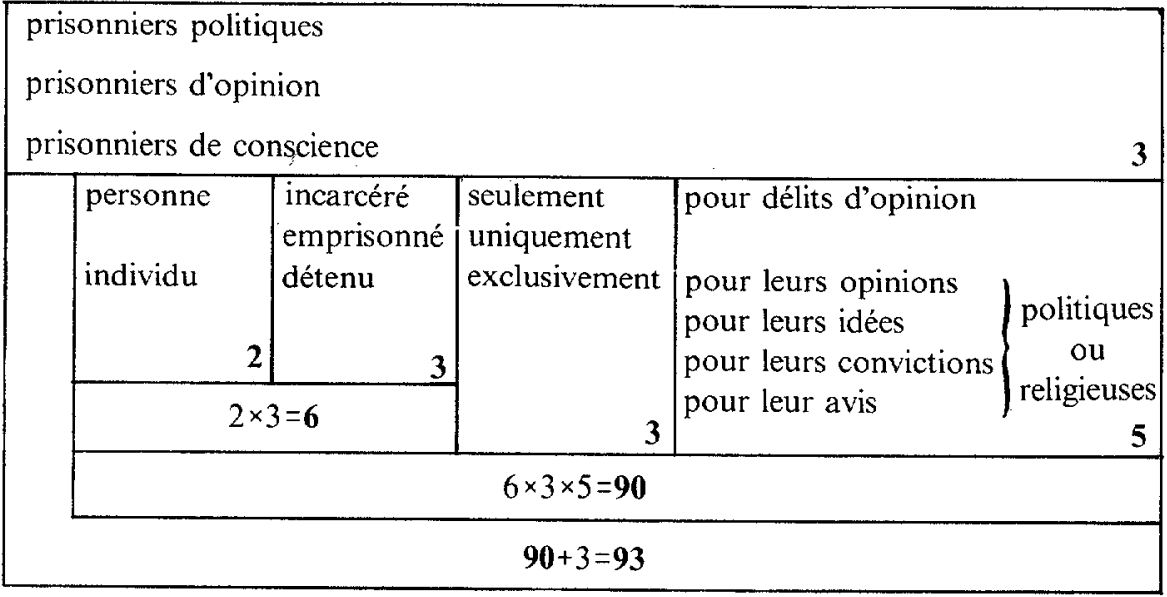


Les phrases $(8)-(10)$ sont des paraphrases assez synonymes qui expriment le sens dè (6) avec une précision suffisante. Cependant, il est clair que ce ne sont aucunement toutes les paraphrases possibles; peut-être, pourrait-on en estimer le nombre? À cette fin, il convient de recenser quelques variantes lexicales existantes pour les "faisceaux" sémantiques figurant dans (6); on va y procéder à l'aide du tableau ( 11 ), au bas de la page opposée.

Continuation de (II)

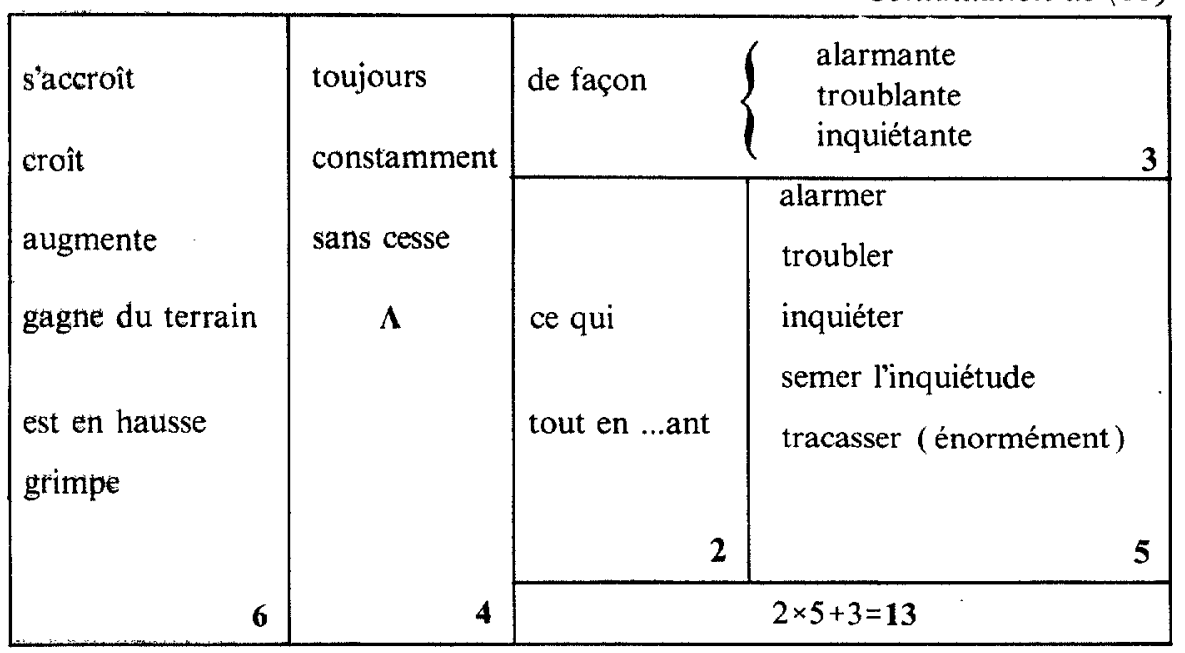

le monde entier tout le monde beaucoup de gens l'opinión publique $\Lambda$ 5
NB1: $\Lambda$ est le symbole d'une chaîne vide; c'està-dire, $\boldsymbol{\Lambda}$ dans une colonne montre que la position correspondante dans la phrase peut être laissée vide.

NB2: le chiffre en gras donne le nombre total de variantes dans sa case.

Une simple multiplication du nombre des variantes suggérées dans ( 11 ) nous donne:

$$
6 \times 7 \times 3 \times 93 \times 6 \times 4 \times 13 \times 5=18280080,
$$

ou environ 20 millions de paraphrases!

Il est vrai que certaines combinaisons de variantes entre elles peuvent se révéler inacceptables; mais, de l'autre côté, on n'a pas tenu compte des variantes suivantes:

(i) ne manque pas d'inquiéter...

$\left.\begin{array}{l}\text { (ii) ne }\left\{\begin{array}{l}\text { laisse } \\ \text { cesse }\end{array}\right\} \text { pas d'inquiéter... } \\ \text { (iii) le nombre de ... s'accroît de façon que }\end{array} \quad \begin{array}{l}\text { on } \\ \text { le monde } \\ \text { tout le } \\ \text { monde }\end{array}\right\}$ est $\left\{\begin{array}{l}\begin{array}{l}\text { troublé } \\ \text { inquiété } \\ \text { alarmé } \\ \text { tracassé }\end{array}\end{array}\right\}$

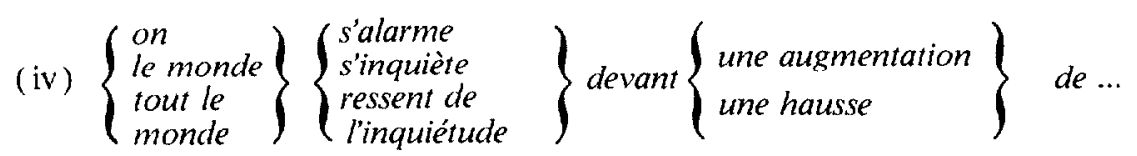


(v) au lieu d'assister à ... on est témoin de ...

et plusieurs autres. On voit donc que le nombre de paraphrases pour un sens donné exprimable dans le cadre d'une phrase peut aller jusqu'à des dizaines de millions. Et si l'on se permet de considérer des énoncés plus longs, comme des paragraphes entiers, le nombre de paraphrases plus ou moins synonymeș devient vraiment astronomique.

Ce fait - l'abondance synonymique des langues naturelles - a une importance primordiale pour le modèle «Sens $\Longleftrightarrow$ Texte». Il en découle que la correspondance DIRECTE entre les sens et les textes est impossible: elle est interdite par la variété immense de textes qu'il faut faire correspondre à un sens donné, en cas général.

On se voit donc contraint à avoir recours à des niveaux intermédiaires ${ }^{7}$ de représentation linguistique ${ }_{2}$. Il semble raisonnable de les choisir en considérant les propriétés les plus saillantes des énoncés linguistiques, qui sont, à mon avis (coïncidant avec le point de vue de la linguistique traditionnelle), d'une part, l'existence d'une organisation hiérarchique en phrase, et d'autre part, l'existence de mots ayant, à leur tour, une organisation interne. Cela nous conduit à deux niveaux intermédiaires:

- le niveau de représentation syntaxique (SyntR)

et

- le niveau de représentation morphologique (MorphR).

Il se trouve, en outre, que ces deux niveaux, ainsi que le niveau phonique, doivent être divisés en deux sous-niveaux chacun: p r of ond (D-, de deep) et de surface (S-), de façon que le tableau définitif ressemble à peu près à ce qui suit:

1. Représentation sémantique (SemR): SENS.

2. Représentation syntaxique (SyntR):

2a. Profonde (DSyntR);

2b. De surface (SSyntR).

3. Représentation morphologique (MorphR):

3a. Profonde (DMorphR);

3b. De surface (SMorphR)

4. Représentation phonique (PhonR):

4a. Profonde, ou phonologique $(\mathrm{DPhonR}=$ PhonolR $)$;

4b. De surface, ou phonétique $($ SPhonR $=$ PhonetR $)$ : TEXTE.

Une représentation profonde est orientée plutôt du côté du sens et une représentation de surface est orientée plutôt vers la forme phonique. En même temps, toutes les représentations énumérées sont des objets gigognes composés — tout comme la SemR (voir plus haut) - d'autres objets formels appelés structures; par exemple:

(12) DSyntR = \{DSyntS, DSynt-CommS, DSynt-AnaphS, DSynt-ProsS $\}$,

où

DSyntS est la structure syntaxique profonde,

DSynt-CommS est la structure syntactico-communicative profonde (se rapportant à l'opposition "le thème vs le rhème ", à la mise en relief, etc.),

DSynt-AnaphS est la structure syntactico-anaphorique profonde (indiquant les coréférentialités, etc.),

et

DSynt-ProsS est la structure syntactico-prosodique profonde (ici, les prosodèmes à charge sémantique doivent être représentés). 
Je ne vais pas justifier ce choix de niveaux et de sous-niveaux de représentation linguistique, (pour les détails voir Mel'čuk 1974a: 31-42) ni caractériser de plus près la composition des représentations proposées; on pourra obtenir quelques renseignements substantiels plus bas, dans la section II. À ce moment-ci, je tiens à souligner que chaque niveau de représentation est, essentiellement, une LANGUE FOR MELLE construite par le linguiste pour chaque langue naturelle étudiée. En d'autres mots, une représentation linguistique ${ }_{2}$ d'un énoncé linguistique ${ }_{1}$ est une expression dans une langue formelle; arriver à un text è à partir d'un sens donné (ou vice versa) veut dire effectuer une série de traductions:

(13) SemR $\Longleftrightarrow$ DSyntR $\Longleftrightarrow$ SSynt $\longleftrightarrow$ DMorphR $\Longleftrightarrow$ SMorphR $\Longleftrightarrow$ DPhonR $\Longleftrightarrow$ SPhonR.

Par conséquent, une langue, ou plutôt, le MODĖLE d'une langue, est un système de mécanismes assurant les traductions mentionnées ci-dessus. Ces mécanismes, ou les composants linguistiques, ${ }_{1}$, seront exemplifiés plus bas.

Remarque. Il ne faut pas croire que les transformations décrites par (13) se produisent en réalité toujours successivement: (13) n'est plus qu'une façon commode de prẻsenter les choses, car normalement tous ces composants linguistiques ${ }_{1}$ sont enchevêtrés et fonctionnent tous à la fois, se nuisant et s'entraidant en même temps, d'après les règles du contrôle central; qu'on peut ignorer pour le moment, puisque ces règles ne relèvent pas de la linguistique comme telle (voir plus bas, page 290).

Donnons maintenant un rapide résumé de tout ce qu'on a dit dans la section I:

(14) On propose de considérer toute langue humaine comme un système de correspondances entre les sens et les textes, ces correspondances étant établies sur quelques niveaux de représentation différents. Un modèle linguistique $1 / 2$ apparaît donc comme un système de traduction entre les représentations linguistiques $1 / 2$. La linguistique devient, dans cette optique, une science de la traduction, l'usage du langage humain constituant un procès de traduction.

Telle est notre conclusion tirée du postulat (3) et ne constituant qu'une reformulation de la thèse (1): il n'y a que de la traduction dans le comportement linguistique, des êtres humains.

\section{MODÈLES LINGUISTIQUES ${ }_{2}$ DU TYPE “SENS $\Longleftrightarrow$ TEXTE»}

Pour donner plus de clarté aux propos de la section I, on va présenter, à titre d'illustration, quelques moyens linguistiques 2 qui semblent nécessaires pour réaliser la synthèse d'une phrase française assez simple - ou plutôt pour l'obtenir comme une traduction de son équivalent anglais.

Je vais m'en tenir aux cadres d'une variante spécifique du modèle linguistique ${ }_{2}$ du type «Sens $\Longleftrightarrow$ Texte », dont l'élaboration a été entreprise et poursuivie pendant 13 années par A. Žolkovskij et le présent auteur: Žolkovskij-Mel'čuk 1967, Mel'čuk-Žolkovskij 1970, Mel'čuk 1974a et 1974b. Il va de soi que les particularités techniques de ce modèle n'ont pas de véritable importance pour mes buts ici. En même temps, il est évident qu'il n'est pas possible soit de donner toutes les explications nécessaires soit de décrire tous les moyens 
indispensables pour la construction de notre phrase échantillon: leur complexité, mêmę dans le cas d'un exemple des plus primitifs, dépasse les limites de cet article.

Soit une phrase anglaise:

(15) I| did not say | they were smart, $\mid$ théy said $\mid$ they were smart;

on prendra (16) comme une des traductions possibles de (15) en français:

(16) $\widehat{\text { Ce n'est pas moi }}|\overparen{\text { qui ai dit }}| \widehat{\text { quills étaient intelligents, }}|| \widehat{\text { ce sont eux }} \mid \widehat{\text { qui le }}$ prétendent.

(Les exemples (15) et (16) sont empruntés à Darbelnet 1971:20.)

On notera qu'il est nécessaire de marquer les prosodies respectives - porteuses de sens: accents emphatiques (marqués par $/$ ), contours d'intonation et pauses (frontières entre les groupes rythmiques); avec des prosodies différentes, les phrases (15) et (16) peuvent avoir un sens différent ${ }^{8}$.

On se pose donc comme tâche de décrire les correspondances entre (15) et (16) en termes d'un modèle linguistique $1 / 2$ du type «Sens $\Longleftrightarrow$ Texte ", en les présentant en deux étapes:

A. Construction d'abord d'une représentation sémantique (SemR) commune pour (15) et (16) - en supposant que les deux phrases sont absolument synonymes, ou expriment le même sens, - - cette SemR constituant le point de départ pour la synthèse de la phrase (16); construction ensuite des représentations intermédiaires entre la SemR de (15) - (16) et la phrase (16) elle-même. (On ne citera pas les représentations respectives

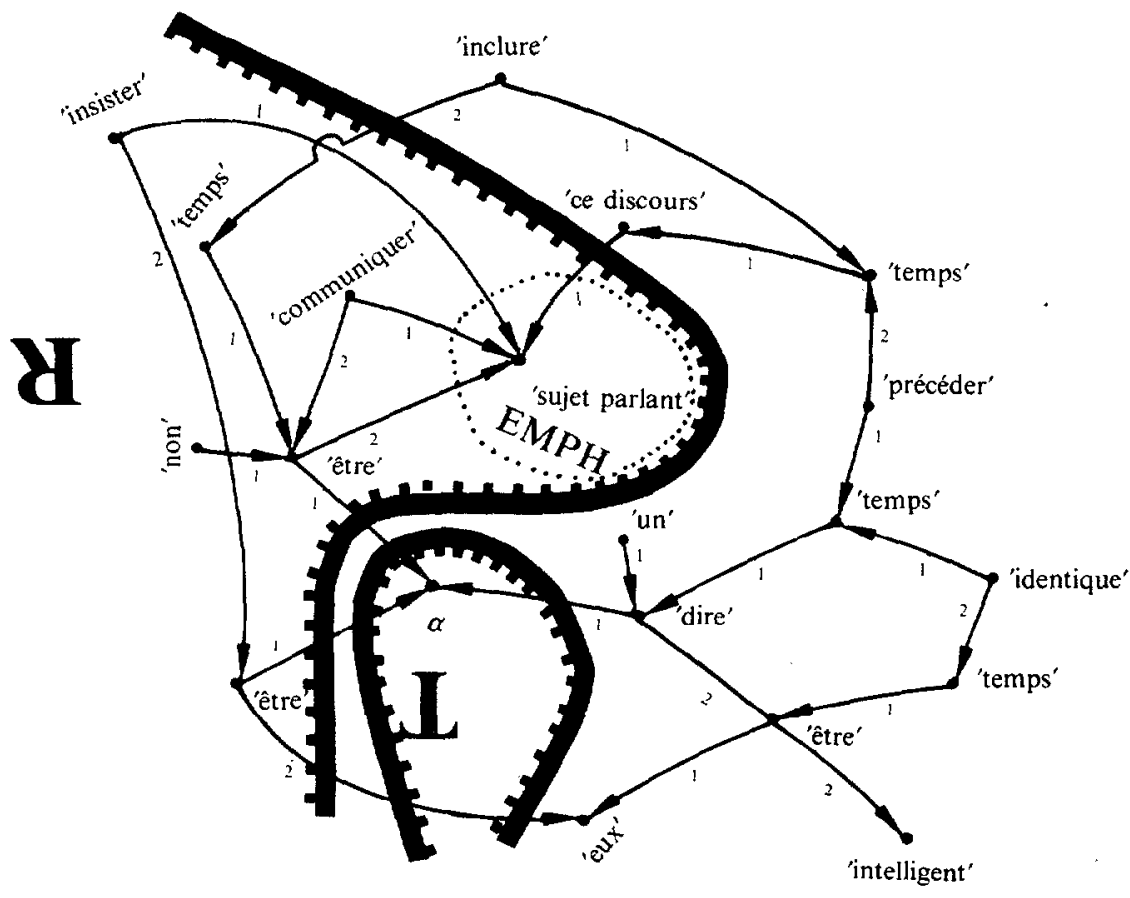


pour ( 15 ), c'est-à-dire, pour la phrase anglaise, afin de ne pas trop disperser l'attention du lecteur.)

B. Construction de règles reliant entre elles toutes les représentations de la phrase (16), ou des com posant $\mathrm{s} \mathbf{d u}$ modè le. Les règles présentées sous $B$ forment un minifragment de la langue française. (De même pour les raisons ci-mentionnées, on ne donnera pas de règles correspondantes de l'anglais: surtout parce que c'est le même type de règles, et les différences spécifiques et idiomatiques ne nous intéressent pas trop dans le présent contexte.)

\section{A. LES REPRÉSENTATIONS LINGUISTIQUES ${ }_{2}$ DE LA PHRASE (16)}

1. La représentation sémantique (SemR): voir (17), page opposée.

Verbalisation approximative de (17):

(18) 'L'auteur de ce discours parle de quelqu'un [ $=\alpha$ ] qui a dit [ = 'une fois et avant le moment du présent discours' ]: «Ils sont intelligents »; il communique emphatiquement qu' $\alpha$ n'est pas lui et insiste qu' $\alpha$ sont eux [les mêmes "eux" qui sont intelligents $]^{\prime}$.

Notons que la configuration des sémantèmes 'un' $[=$ 'une fois' $]$ et 'le temps de dire précède le temps de ce discours' traduit la signification du passé composé dans (16) (et du Past Tense anglais dans (15)).

2. La représentation syntaxique profonde (DSyntR).

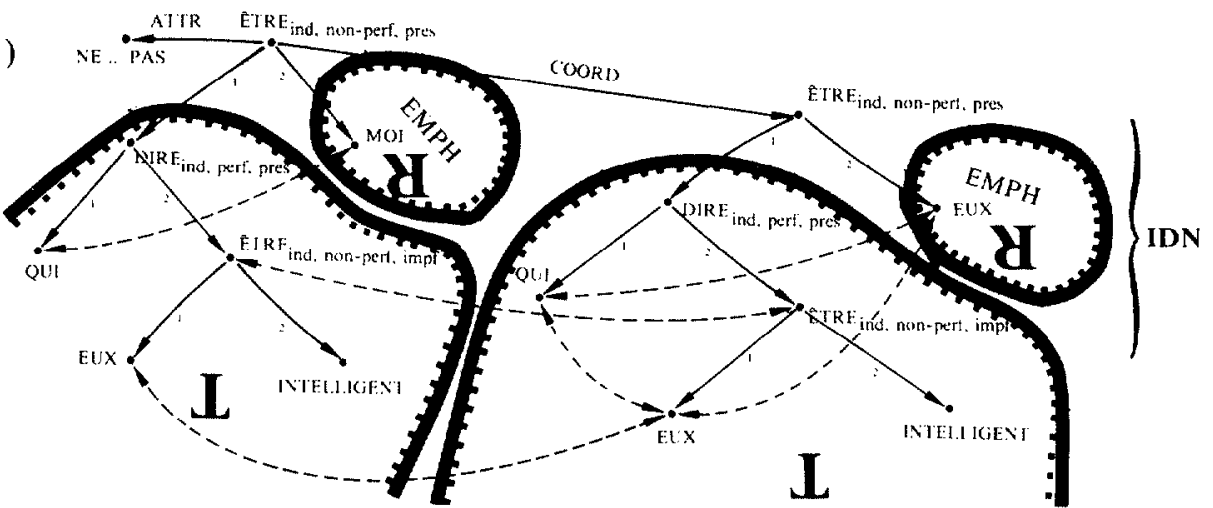

La DSyntR consiste en quatre structures (comparez la page $278,(12)$ ):

Structure syntaxique profonde qui est un arbre de dépendances dont les nœuds ne sont pas ordonnés linéairement. Chaque nœud représente un lexème plein de la phrase (aucun lexème outil, c'est-à-dire, sémantiquement vide, n'est représenté). Chaque branche représente une relation syntaxique profonde (universelle): COORD(inative), ATTR(ibutive), et les relations prédicatives - entre un mot-prédicat et ses arguments marquées par des chiffres $(1,2, \ldots)$.

Structure syntactico-communicative profonde, où le thème et le rhème sont identifiés, tout comme la mise en relief, etc.

Structure syntactico-anaphorique profonde: les flèches pointillées à deux têtes relient les nœuds coréférentiels ( $=$ se référant au même objet réel). 
Structure syntactico-prosodique profonde: l'abbréviation IDN (= intonation déclarative neutre) montre qu'il s'agit d'une phrase déclarative neutre - sans ironie, sans menace, ni moquerie, etc.

Comme on voit, une DSyntR présente les aspects différents de la phrase en question strictement séparés - par quatre structures différentes.

Il faut avouer, cependant, que (19) n'est pas encore la DSyntR de la phrase (16), qui est notre objectif: (19) détermine une phrase synonyme à (16), à savoir,

(16') Ce n'est pas moi qui ai dit qu'ils étaient intelligents, ce sont eux qui L'ONT DIT

(la partie différente est imprimée en majuscules).

Mais, comme on sait très bien que le français déteste les répétitions lexicales (c'est presque une loi grammaticale), il semble naturel d'avoir dans une bonne description du français une règle-filtre qui, en cas de répétition lexicale dans une DSynt $R$, met cette dernière au rebut. À la suite de cette règle, une autre DSynt $R$, dérivée parallèlement de la même Sem $R$ mais ayant une des occurrences répétées remplacée par un lexème synonyme ou quasi synonyme, aura la priorité. Somme toute, on aura pour (19) une DSyntR variante:

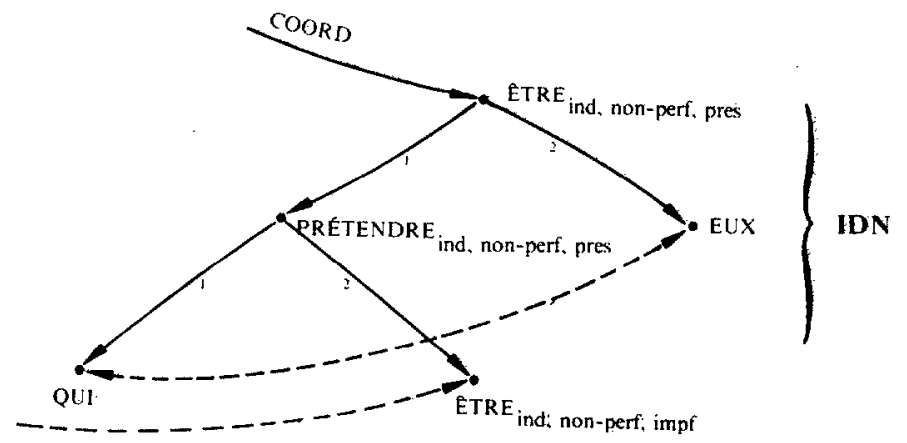

tout le reste étant identique à (19).

Notons que l'indice morphologique du mode indicatif que portent les deux ETRE en haut de la structure (19) ou (19') traduit le sous-graphe sémantique 'le sujet de ce discours communique que ...', tandis que le même indice attaché aux deux ETRE inférieurs est conditionné par leur dépendance des verbes DIRE et PRETENDRE. Les indices de "perf" et "pres " combinés (= passé composé) que porte DIRE dans (19) traduisent l'indication de la SemR que 'le temps de ce dire précède le temps de ce discours', etc.

3. La représentation syntaxique de surface (SSyntR): voir (20), page suivante.

La SSyntR est constituée des mêmes genres de structures que la DSyntR. Les. différences les plus importantes sont les trois suivantes:

1) La structure syntaxique de surface inclut tous les mots de la phrase représentée même les mots outils, tels que prépositions et conjonctions régies, sujets apparents (dummy subjects) comme ce ou il, etc., qui sont absents de la DSyntS. 


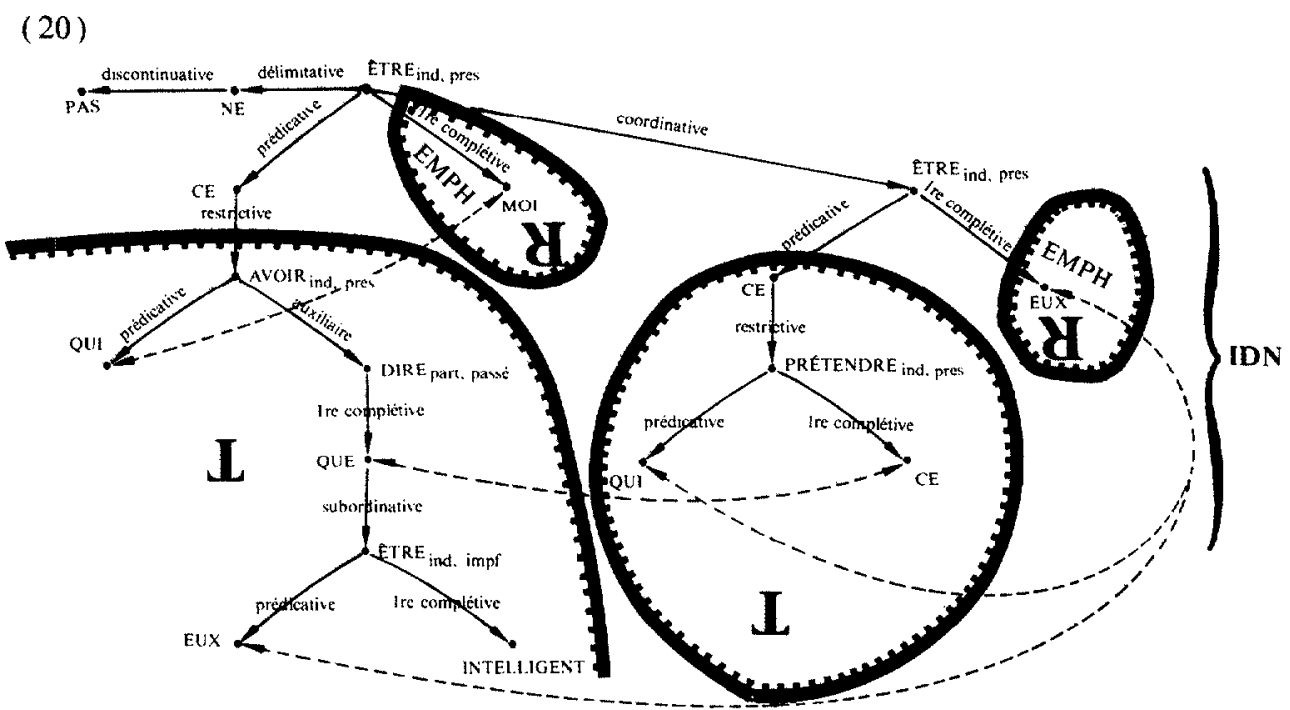

2) Les branches de la SSyntS sont étiquetées avec des symboles de relations syntaxiques de surface (SSyntRel), qui sont spécifiques pour chaque langue et qui en identifient les constructions syntaxiques.

3) Les sousarbres susceptibles de pronominalisation sont remplacés par des pronoms (ou des clitiques) correspondants: ainsi, le sousarbre [ Ils prétendent] qu'ils sont intelligents est remplacé par le pronom $\mathrm{CE}$ (dont la forme objectivale directe est le: Ils le prétendent). note 9 .

À propos de l'analyse des relatives emphatiques qui ai dit...et qui le prétendent, voir

Les SSynt-CommS, SSynt-AnaphS et SSynt-ProsS ressemblent à ses vis-à-vis profonds de telle façon qu'on n'est pas obligé de donner ici des explications particulières.

4. La représentation morphologique profonde.

(21) $\mathrm{CE}_{\text {suj }}$ NE ETRE $E_{\text {ind. pres. 3sg }}$ PAS $\mathrm{MOI}_{\text {ton }}{ }^{\prime} 1$

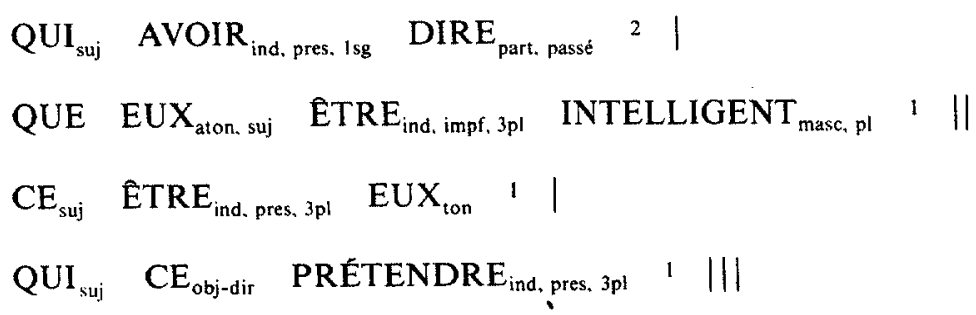

On arrêtera ici, les représentations des niveaux supérieurs n'ayant pas de portée particulière vis-à-vis du problème qui nous occupe. 


\section{B. LES RĖGLES DE CORRESPONDANCE ENTRE \\ LES REPRÉSENTATIONS DE NIVEAUX VOISINS: \\ LES COMPOSANTS DU MODËLE LINGUISTIQUE ${ }_{2}$}

Maintenant, on peut aborder les règles qui constituent, prises ensemble, le modèle linguistique ${ }_{2}$. Comme il est peu commode de citer toutes les règles indispensables, ne serait-ce que pour le passage (17) $\Longrightarrow(19) /\left(19^{\prime}\right) \Longrightarrow(20) \Longrightarrow(21)$ nous n'en donnerons ici que quelques-unes pour chaque étape.

(i) Règles sémantiques: SemR $\longleftrightarrow$ DSyntR, ou le composant sémantique du modèle linguistique $_{2}$

$$
\operatorname{Reg}^{\text {SEM }} \text { 1: }
$$
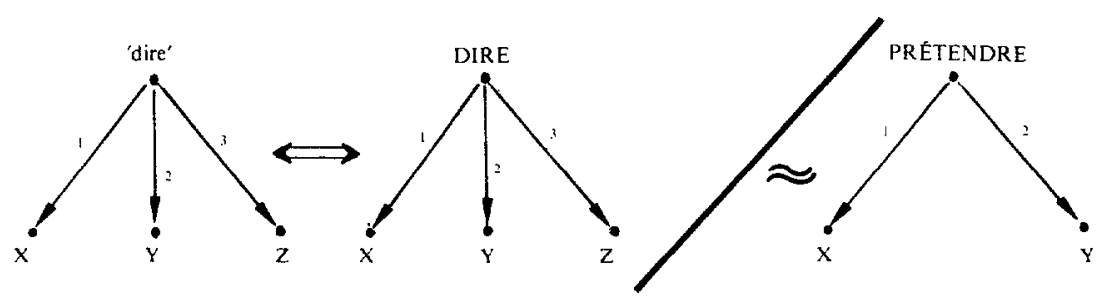

$\operatorname{Reg}^{\mathrm{SEM}} 2$ :
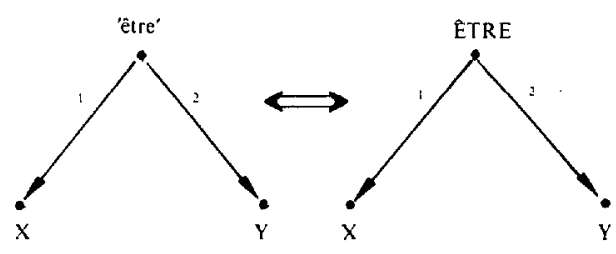

$$
\operatorname{Reg}^{S E M} \quad 3:
$$

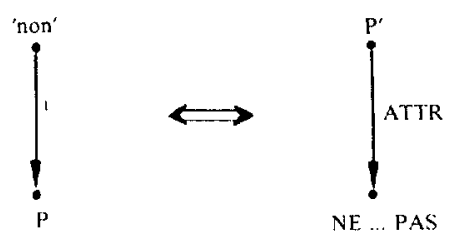

$\mathrm{P}^{\prime}$ dénote l'image syntaxique profonde du nœud (ou sousgraphe) sémantique $\mathrm{P}$, c'est-àdire le lexème correspondant à $P$.

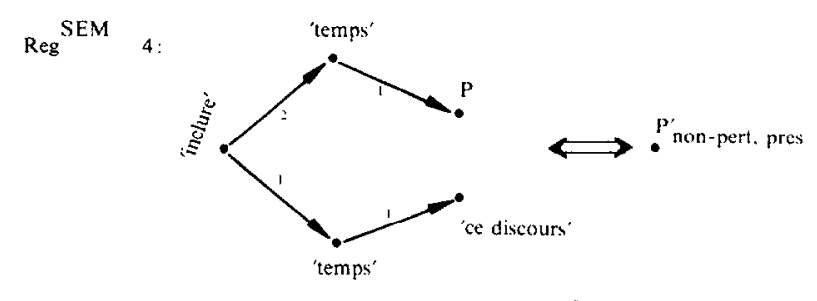



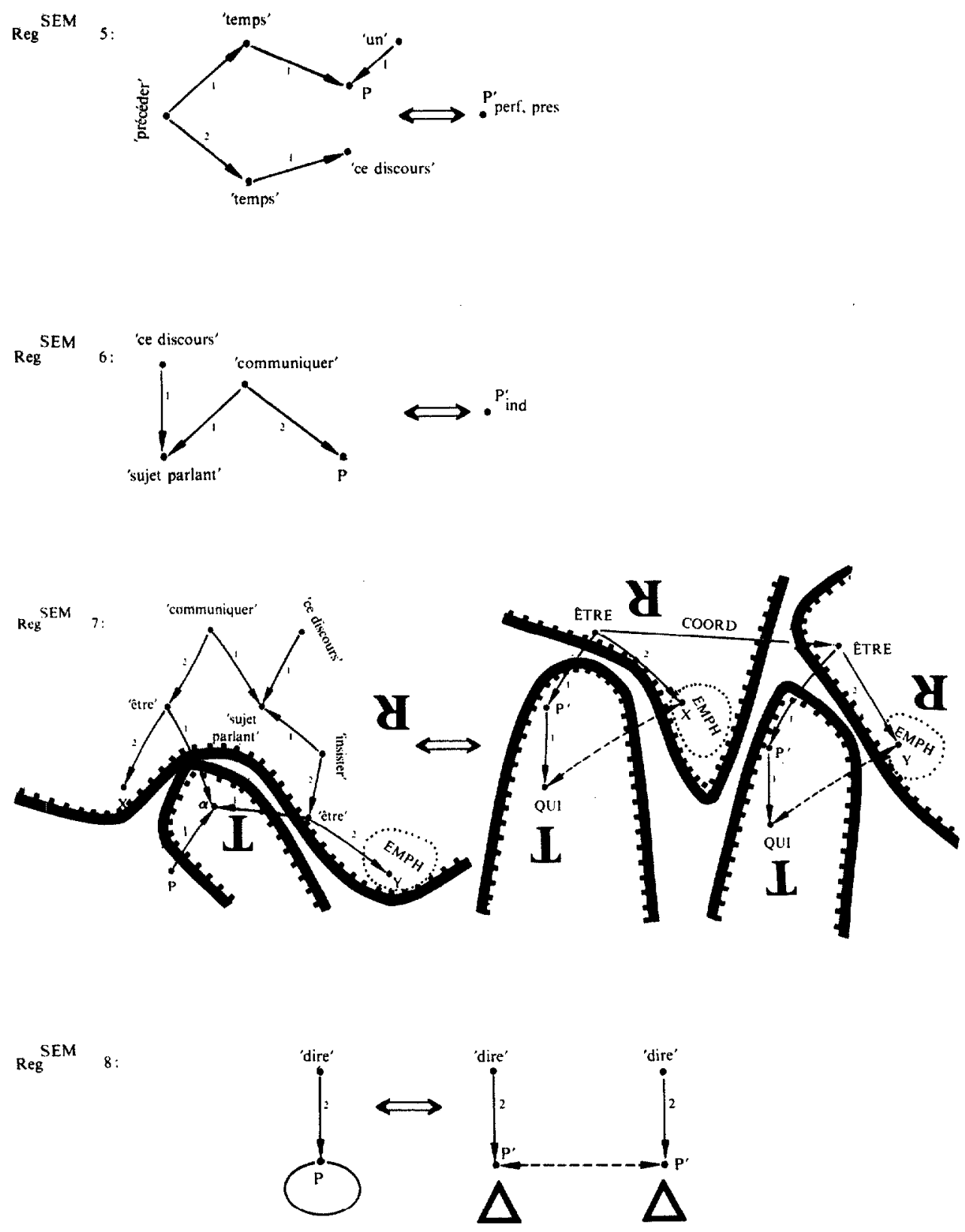

(P) dénote un sousgraphe sémantique ayant pour représentant le nœud P ;

$\mathrm{P}^{\prime} \dot{A}$ dénote un sousarbre de dépendance avec la racine $\mathrm{P}^{\prime}$. 


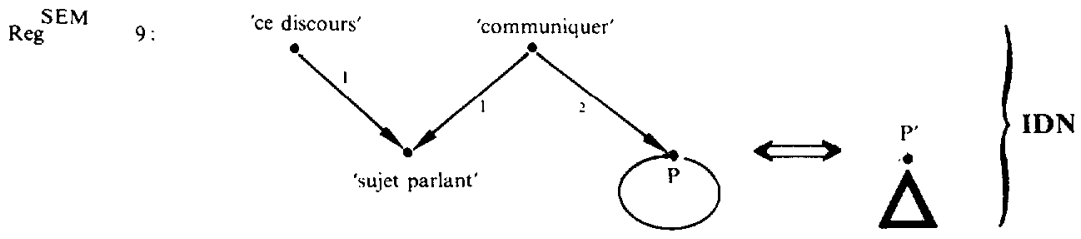

NB: il ne faut pas prendre ces règles (comme, d'ailleurs, toutes les autres règles données plus bas) trop au sérieux. Elles sont écrites sous une forme très approximative et grossière (sans décomposition nécessaire du sémantème 'dire' en Reg $\operatorname{SEM} 1$, sans généralisation évidente en RegSEM 7, etc.) avec un seul but: donner au lecteur une idée générale.

Les règles RegSEM $1-3$ sont des règles $\mathrm{sé} \mathrm{m}$ a $\mathrm{n}$ t i co-l ex i ca le $\mathrm{s}$, ou bien des entrées du dictionnaire: elles s'occupent des correspondances entre des sousgraphes sémantiques et des lexèmes français porteurs du même sens.

Les règles RegSEM 4-6 sont des règles sémantico-morphologiques qui établissent les correspondances entre les sousgraphes sémantiques et des catégories grammaticales françaises: présent ( $\operatorname{Reg}^{\mathrm{SEM}} 4$ ) signifie, entre autres, la coïncidence $d u$ temps de l'événement décrit avec le moment du discours; etc.

La règle RegSEM 7 est une règle sé $\mathrm{mantic} 0$-c o $\mathrm{mmunic}$ a tive, responsable de la correspondance entre l'organisation communicative du graphe sémantique (thème $v s$ rhème,...) et le schéma général de la DSyntR correspondante: la distribution du thème et du rhème, le choix de la construction globale pour mieux rendre le message contenu dans la SemR, etc.

La règle $\mathrm{Reg} \mathrm{SEM} 8$ est une règle sé $\mathrm{man}$ tic $\mathrm{o}$-a $\mathrm{naph}$ o rique, et la règle 9 est une règle sémantico-prosodique: on a présenté ici tous les types de règles sémantiques (sauf les règles sémantico-syntaxiques qui prévoient les constructions syntaxiques à chargement sémantique: ce genre de construction est rare en français).

(ii) Règles syntaxiques: DSynt $\mathrm{C} \Longleftrightarrow \mathrm{DM}$ orph $\mathrm{R}$, ou le composant syntaxique du modèle linguistique ${ }_{2}$

a) Syntaxe profonde: DSyntR $\Longleftrightarrow$ SSyntR

Reg DSynt I:

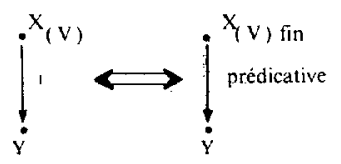


Seul, un verbe fini (= tensed verb) peut être membre gouverneur dans une relation prédicative. Pour les raisons d'économie d'espace, on a omis les conditions assez compliquées pour l'emploi des verbes finis en français.

$$
\text { Reg DSynt 2: }
$$




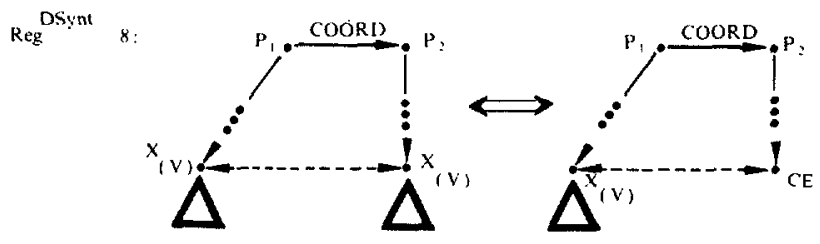

Les règles RegDSym $1-4$ sont des règles syntaxiques à proprement parler: elles établissent des correspondances entre les constructions syntaxiques profondes et celles de surface en introduisant, là où il le faut, des mots outils ( $\operatorname{Reg} \operatorname{DS}_{y n t} 3:$ QUE), etc.

La règle $R e g{ }^{D S y n t} 5$ décrit les formes dites analytiques (en ce cas-ci, le passé composé), et la règle $\operatorname{Reg}^{\mathrm{Ds} y n t} 6$ - les mots discontinus (ne ... pas, ne ... que,...).

La règle RegDSym 7 est un règle syntactico-communicative qui nous dit, en gros, que la division en thème $\sim$ rhème, emphatisé $\sim$ non-emphatisé, etc. est maintenue entre la DSyntR et SSyntR.

Enfin, la règle RegDsynt 8 est une règle de pro nominalis ation: un sousarbre verbal $X_{(v)}$ qui est lexicalement identique et coréférentiel à un autre sousarbre et qui se trouve, en plus, dans un contexte arborescent indiqué dans la règle en question, est remplacé par le pronom CE (le dans Ils le prétendent est considéré comme forme objectivale directe de $C E$, voir plus haut).

b) Syntaxe de surface: SSyntR $\Longleftrightarrow$ DMorphR

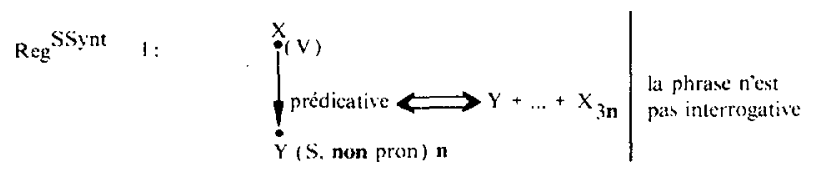

La variable $\mathbf{n}$ dénote le nombre grammatical, de sorte que $\operatorname{Reg}^{\text {ssynt }} \mathbf{I}$ décrit l'accord en nombre du verbe avec le sujet (dans le cas général).

$$
\begin{array}{ll|l}
\text { RegSSynt 2: } & \mid \begin{array}{l}
\text { Si } Z=(S), \text { non pron }) . \\
\text { alors } p(X)=3: \\
\text { Si } Z=(S, \text { pron }) \\
\text { alors } p(X)=p(Z)
\end{array}
\end{array}
$$

Reg SSynt 2 prévoit un cas spécial de l'accord «sujet-verbe» en français: si le sujet grammatical est le pronom relatif QUI, le verbe s'accorde en personne et en nombre avec l'antécédent de QUI (c'est-à-dire, avec le nom ou pronom coréférentiel à ce QUI): l'homme qui est ... vs les gens qui sont ... vs moi qui suis ... etc. 


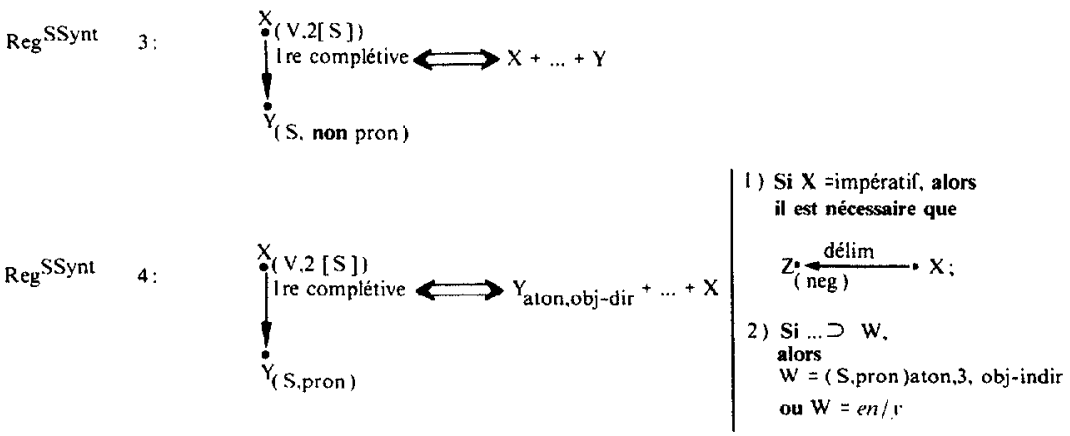

Regssynt 3 et 4 décrivent les constructions « verbe transitif - complément d'objet direct ": un nom dans Regssynt 3, un clitique dans Regssynt 4. La condition 1 dans la dernière spécifie que la précédence du clitique avec des formes verbales impératives n'est possible que si cet impératif est négatif; la condition 2 établit les possibilités de séparer le clitique - objet direct et le verbe par d'autres clitiques seulement, tel que lui, leur, en, y (II m'en tire, Il la leur recommande etc.). ${ }^{10}$

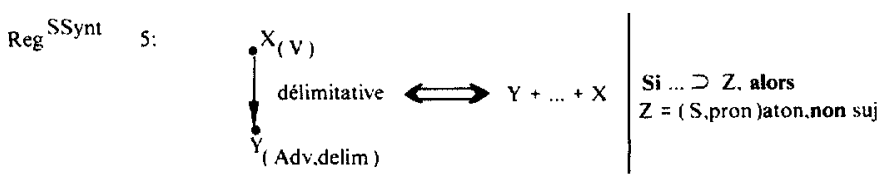

Je ne $[\mathrm{Y}]$ la $[\mathrm{Z}]$ reyarde $[\mathrm{X}]$ pas.
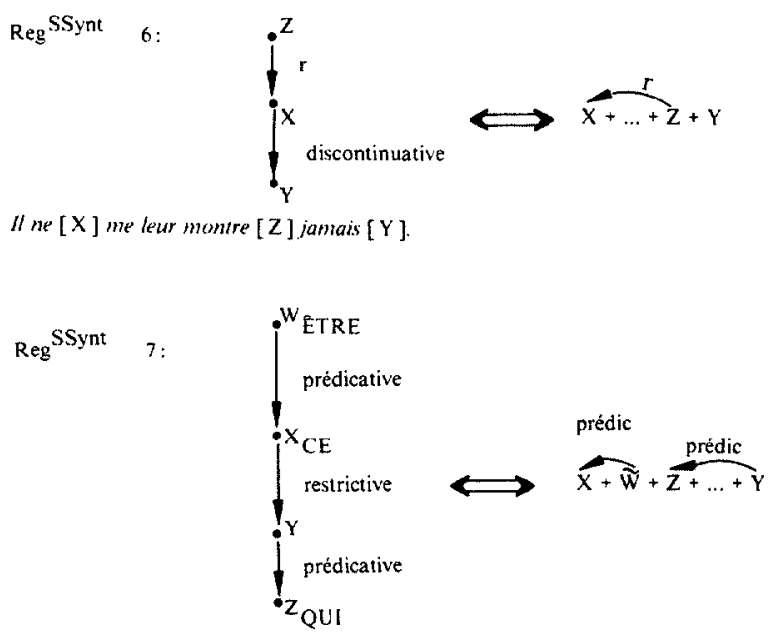

$\mathrm{Ce}[\mathrm{X}]$ n'était $[\mathrm{W}]$ aucunement Claudia Cardinale qui $[\mathrm{Z}]$ avait $[\mathrm{Y}]$ produit tout ce désordre.

Ici, $\widetilde{\mathrm{W}}$ signifie un sousarbre complet — sauf $\mathrm{X}$ et tous ses dépendants - ayant $\mathrm{W}$ pour racine. 


$$
\text { RegSSynt } 8:
$$

Les règles Regssyn 1-7 sont des règles syntaxiques de surface sensu stricto: elles assurent les correspondances entre la SSynt $R$ et la structure morphologique profonde de la phrase, c'est-à-dire, la chaîne ordonnée de lexèmes avec morphologisation complète (toutes les variables grammaticales spécifiées).

Les règles Regssynt 8 et 9 sont des règles syntactico-prosodiques: à des configurations déterminées dans les arbres syntaxiques de surface, elles font correspondre les pauses et les contours d'intonation qui sont syntaxiquement conditionnés (comparez la note 8 ). D'autres règles syntactico-prosodiques (que je ne cite ici faute d'espace) dérivent les prosodies de surface à base de la SSynt-ProsS — en d'autres mots, d'après les prosodies sémantiquement chargées (question, étonnement, ironie, ...).

Si on continue la liste des règles dont on a énuméré environ deux douzaines, on peut espérer obtenir, au bout du compte, un modèle complet de la langue française.

Le lecteur a déjà remarqué, sans doute, une propriété très importante de ces règles: ELLES NE SONT PAS ORDONNÉES. Il est clair que dans une procédure réelle de passage de SemR à DSyntR (ou vice versa) etc., les règles nécessaires doivent être appliquées dans un ordre qui est loin d'être arbitraire ou fortuit. Mais j'insiste sur le fait que la tâche de trouver un ordre optimal (ou du moins raisonnable) pour l'application des règles linguistiques ${ }_{2}$ ne relève aucunement de la linguistique comme une science du langage: c'est un problème assez général des mathématiques ou d'autres sciences (portant sur la conduite intelligente des êtres humains) puisque les mêmes lois doivent gouverner le choix d'un ordre déterminé des règles pour n'importe quelle variété du comportement humain. Le linguiste en tant que tel doit se satisfaire de l'élaboration d'une liste exhaustive de règles linguistiques, telles qu'un ordre d'application puisse exister en principe: cela peut être, par exemple, un tri complet de toutes les règles (ce qui, naturellement, ne vaut pour aucune tâche pratique). 
Récapitulons: pour exprimer le sens (17) (page 280) en français de même que pour comprendre la phrase française (16) (page 280) il faut avoir à sa disposition un ensemble de règles illustré par les règles citées sous B (pages 284-290). Des règles de ce genre sont nécessaires pour exprimer ce même sens en anglais ou pour comprendre la phrase anglaise (15). Et ces deux ensembles de règles sont nécessaires (bien qu'aucunement suffisants) pour traduire (15) par (16) ou vice versa. Cela veut dire seulement que:

(22) la traduction "authentique», la traduction INTER-linguistique ${ }_{1}$, c'est-à-dire effectuée entre deux langues naturelles, se compose toujours au moins de deux traductions INTRA-linguistiques ${ }_{1}$ :

(i) la traduction entre le texte (dans la langue de départ) et le sens de ce texte;

(ii) la traduction entre le sens du texte (dans la langue d'arrivée) et ce même texte.

NB: très souvent, une troisième traduction est nécessaire, à savoir, une transformation du sens de départ dans le sens d'arrivée. Cette complication a lieu fréquemment si le sens du texte de départ ne peut pas être exprimé d'une façon adéquate dans la langue d'arrivée. Alors on est forcé de trouver un autre sens, le plus proche possible du sens donné et exprimable avec une certaine facilité dans la langue vers laquelle on traduit. Pourtant, ladite transformation n'est guère linguistique ${ }_{2}$ : elle relève de la logique, des connaissances encyclopédiques, du know-how humain et de beaucoup d'autres choses aussi, mais pas du tout des langues impliquées. Cela nous permet d'en faire abstraction dans le cadre de cet exposé.

La conclusion (22) n'est pas originale: plusieurs spécialistes de la traduction l'ont déjà faite et répétée à maintes reprises (par exemple, Malblanc 1961: 18-19 ou Nida 1975: 79-80'1). Il faudrait enfin qu'elle soit acceptée par la linguistique, en théorie et en pratique, et que des systèmes translatifs, ou transductifs, du type esquissé plus haut, soient adoptés pour la description des langues naturelles.

\section{PROBLEMES TYPIQUES DE TRADUCTION - PROBLĖMES DE LINGUISTIQUE GÉNÉRALE}

Avant de passer à des remarques finales, j’aimerais analyser ici quatre classes de problèmes généralement considérés comme caractéristiques justement du domaine de la traduction. J'espère pouvoir montrer qu'en réalité ce sont des problèmes de linguistique tout simplement et qu'ils doivent être traités et résolus dans toute description monolingue de n'importe quelle langue naturelle.

(i) Problèmes de compréhension: le traducteur éprouve des difficultés parce qu'il ne comprend pas le texte de départ. Exemple: angl. brown shoe admiral, qui a été un cassetête pour un traducteur français (Nakos-Aupetit 1977: 261-262). Cependant, ici il suffit tout simplement de connaitre le jargon militaire américain: brown shoe = 'aviateur naval' et, par conséquent, brown shoe admiral = 'amiral des forces aéronavales'. Rien de spécifique, relevant exclusivement de la traduction: la cataloguisation de l'expression idiomatique brown shoe (avec une caractérisation détaillée de son usage) concerne avant 
tout la description monolingue de l'anglais. Autant que je puisse juger, il en est de même avec toutes les difficultés de compréhension.

(ii) Problèmes d'expression: la traduction s'avère souvent difficile dans les cas où un sens quelconque est exprimé dans la langue d'arrivée d'une façon tout à fait différente de la langue de départ. Plus précisément, il s'agit ici de la distribution radicalement différente des unités sémantiques parmi les unités lexicales et constructions syntaxiques des deux langues. Les exemples abondent; en voilà quatre, pris plus ou moins au hasard.

- Le boulou (une langue du Cameroun) n'a pas de terme pour 'prépuce' mais un membre viril circoncis ('sans prépuce') et un membre non circoncis ('avec prépuce') sont désignés dans cette langue par des noms différents. Cela a conduit le traducteur de la Bible en boulou à rendre la phrase parlant de 'ramener les prépuces des Philistins' par un phrase en boulou qui veut dire, littéralement, 'ramener les membres virils non circoncis des Philistins' (Belinga 1977: 193-194). Une erreur de traduction? Pas du tout. Nous y voyons une erreur d'expression. Comme il est peu probable que le traducteur n'ait pas compris le sens de la phrase-source, il reste à supposer qu'il s'est exprimé d'une façon maladroite; et un tel faux-pas relève entièrement de la sémantique monolingue.

- Le verbe anglais to affect a de nombreux équivalents français, et il est parfois pénible de sélectionner celui que le contexte réclame:

(23) areas affected by the storm = régions éprouvées par la tempête;

affect the safe operation = nuire à la sécurité;

person affected by the noise = personnes incommodées par le bruit;

Our way of life has been profoundly affected by electricity = L'électricité $a$ bouleversé notre mode de vie; etc. (Delisle 1977:277).

Pourtant, la difficulté ne devient pas moindre si on se place du point de vue de la synthèse "pure » du français, en laissant la traduction complètement hors du jeu: même pour celui qui décrit le français sans égard à l'anglais ou à d'autres langues, il est toujours indispensable d'indiquer comment on doit exprimer en français l'idée de 'l'action d'une tempête sur une région' (c'est éprouver ou ravager), 'l'action du bruit sur des personnes' (incommoder), 'l'action négative de n'importe quoi sur la sécurité' (nuire à), etc. C'est une tâche dont il est, peut-être, plus facile de se rendre compte en traduisant - mais qui est, elle aussi, purement monolingue. Pour la résoudre, il faut présenter une description de la distribution - en fonction du contexte lexical - des lexèmes destinés à exprimer le même sens; dans notre cas, c'est 'l'action de...sur...'12

- En russe, il y a beaucoup de verbes qui désignent des actions et des états assez spécifiques et qui n'ont pas d'équivalents directs en français. Par ex., probirat'sja = 'se déplacer en surmontant des obstacles ou essayant de ne pas être aperçu' (dans l'obscurité, dans une foule, en se cachant, etc.) ou sdërnut' = 'arracher ou décrocher d'une façon brusque et négligée'. Pour rendre en français les phrases russes qui contiennent ces verbes, il faut trouver des moyens linguistiques, qui puissent exprimer le sens perdu à cause des verbes français plus neutres s'avancer (suivre, se déplacer, ...) ou décrocher tenant lieu de probirat'sja et sdërnut'; on peut utiliser des compléments circonstanciels:

(24) Otec probiralsja za nim [on sait par le contexte précédent que le père ne voulait pas être vu ou entendu de son fils ] 


\section{(24') Son père le suivait à pas de loup;}

ou encore:

(25) Ona sbežala vniz, sdërnula s vešalki plašč $i$ vybežala na ulicu

(25') Elle descendit en hâte, décrocha son imperméable et sortit,

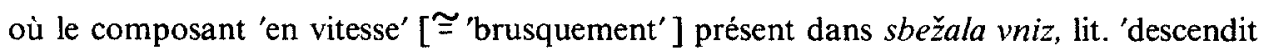
[l'escalier ] en courant', sdërnula et vybežala 'sortit en courant' est représenté en français une seule fois - par en hâte. (Les exemples sont adaptés de Gak 1977: 107-109.)

Certes, des cas comme ceux-là sont le cauchemar des traducteurs. Peut-on, pourtant, dans une description scientifique du lexique français, ou plus généralement, de la sémantique française, passer sous silence un fait aussi fondamental que celui de l'absence de verbes plus spécifiques dotés d'un sens qui inclut des indications sur la façon dont l'action est effectuée? Non seulement il faut absolument le faire, mais encore est-il également indispensable de formuler, pour le français, les règles qui ne nous obligent pas à exprimer le «comment" de l'action qu'une seule fois - par un complément circonstanciel de mode ou de façon. En russe, d'autres règles sont en vigueur: le russe préfère l'accord sémantique, de sorte que, si un verbe de la phrase contient le composant sémantique 'en hâte', il est désirable que tous les autres verbes de la même phrase le contiennent aussi (sbežala 'descendit en hâte' - sdërnula 'décrocha brusquement et négligemment' vy'bežala 'sortit en hâte').

- Enfin, le japonais, comparé aux langues européennes, donne un exemple excellent de redistribution de sens. Pour 'j'ai voulu y aller' on dit en japonais

(26) ikitaku narimasita,

où l'on $\mathrm{a}$ :

$i k(i)$ - radical du verbe 'aller';

-ta(i) - suffixe de l'adjectif déverbatif signifiant 'voulant .../ désirant ...';

$-k u$ - suffixe de l'adverbe déadjectival;

nar - _ radical du verbe 'devenir = commencer à être';

-imas $(u)$ - suffixe de la forme finie (formelle) du verbe;

-ita - suffixe du passé.

Littéralement, (26) est représentable comme

(26') 'aller-voul-emment [je] devins'.

Ce qui est exprimé par un suffixe adjectival en japonais est un verbe indépendant en français: - tai = vouloir ce qui est exprimé par une forme grammaticale en français est un verbe indépendant en japonais: le passé composé, exprimant, dans ce cas-ci, le commencement (j'ai voulu $\approx$ 'j'ai commencé à vouloir'), correspond à $n a r-(u)$ au passé; etc.

La divergence entre les deux techniques pour exprimer le même sens est extrême, et cela peut causer des problèmes au cours d'une traduction du japonais en français (ou vice versa). Mais l'essence de ces problèmes - si le texte japonais est bien compris - reste toujours la même: il faut savoir s'exprimer en français, traduction ou pas.

Tous les quatre cas analysés montrent clairement que la distribution différente de 
sens parmi les unités lexicales des langues intéresse la linguistique comme telle; il n'y existe rien qui puisse être qualifié comme relevant exclusivement de la traduction.

(iii) Problèmes d'explication de réalités nouvelles: il est difficile de traduire un texte qui parle de choses inconnues des témoins de la langue d'arrivée.

Voici un bon exemple. En traduisant la Bible du français en boulou, le traducteur s'est heurté à des obstacles tels que chameau, désert (les Boulous ne connaissent que la forêt équatoriale), tentes, ou neige: les objets désignés par ces mots n'existant pas dans l'univers des Boulous, les équivalents lexicaux sont absents de leur langue (Belinga 1977). On est obligé donc de recourir aux emprunts avec explications en notes (chameau, neige), ou bien aux périphrases: désert $=n k \hat{o} t$ si 'terre sans eau', tentes $=$ menda bindélé 'maisons faites avec de la toile', etc. (qui, d'ailleurs, doivent être aussi expliquées par des notes - vu qu'un Boulou ne peut pas, par exemple, s'imaginer un terrain sans eau).

Cependant, les mêmes difficultés se retrouvent quand, loin de toute traduction, on doit décrire en boulou la vie des autres pays et des autres civilisations. L'erreur amusante commise par le traducteur de la Bible en boulou: laine qui a été traduit comme mevul'poils du pubis de la femme' - n'a rien à voir avec la traduction à proprement parler. Une erreur pareille est bien possible chez un Boulou monolingue qui décrit les moutons vus pour la première fois ou les vêtements de laine.

Le problème d'assimilation des nouvelles notions dans une langue - par la voie d'emprunt lexical, de calque lexical ou de création de nouvelles unités lexicales à base de matériel authochtone - est, sans doute, un des problèmes les plus importants de la description linguistique ${ }_{2}$ monolingue. $\bar{A}$ mon avis, on ne saurait trop insister sur le fait que ce problème relève de la linguistique, sa relation logique avec la traduction n'étant guère plus intime que celle de la syntaxe ou de la morphologie de la langue d'arrivée.

(iv) Problèmes d'intraduisibilité. On se demande, depuis des siècles si, vraiment, on peut toujours traduire n'importe quel texte - ou au moins la plupart des textes qu'on vous offre, ou, dans la pire des hypothèses, seulement quelques textes?

Les uns répondent: Non, jamais; rien n'est traduisible. Par exemple, Wilhelm von Humboldt, qui écrit: "Alles Übersetzen scheint mir schlechterdings ein Versuch zur Auflösung einer unmöglichen Aufgabe», se voit appuyé par Ortega y Gasset: "¿ No es traducir, sin remedio, un afán utópico? " (dans « Miseria y esplendor de la traducción »). On peut multiplier les citations sans fin, en évoquant Dante, Benedetto Groce, Gœthe, etc.

Les autres (pas moins nombreux) répondent: Oui, toujours; tout est traduisible. E. Sapir affirme que "... any concept, whether or not it forms part of the system of grammatical categories, can be conveyed in any language ", soutenu en cela par R. Jakobson, qui écrit: "All cognitive experience and its classification is conveyable in any existing language». Et enfin, l'existence d'une armée de traducteurs professionnels, de bureaux et d'organismes s'occupant de la traduction de milliers de textes en des centaines de langues, prouve d'une façon ostensible que plusieurs sont convaincus de la traduisibilité universelle.

Sans doute, on peut trouver des points de vue intermédiaires: il y a des gens qui croient qu'on peut traduire beaucoup de choses, mais pas tout; que l'intraduisibilité existe — à un certain degré - partout, mais qu'elle est loin de tout embrasser; etc. 
Pour prendre position face à ce problème de la traduisibilité, il faut d'abord analyser la notion même de traduisibilité, cf. Wierzbicka (sous presse).

Soient deux langues $L_{1}$ et $L_{2} ; T\left(L_{1}\right)$ et $T\left(L_{2}\right)$ sont des textes quelconques dans ces langues, ' $T\left(L_{1}\right)^{\prime}$ et ' $T\left(L_{2}\right)$ ' étant les sens de ces textes.

(27) On dira que $T\left(L_{1}\right)$ est une traduction exacte de $T\left(L_{2}\right)$

si et seulement si:

(i) ' $\mathrm{T}\left(\mathrm{L}_{1}\right)^{\prime}=$ ' $\mathrm{T}\left(\mathrm{L}_{2}\right)^{\prime}$ [ les deux textes ont le même sens ]

et

(ii) $T\left(L_{1}\right)$ est composé d'unités linguistiques, qui jouent à l'intérieur de la langue $\mathrm{L}_{1}$ le même rôle que les unités composant $T\left(\mathrm{~L}_{2}\right)$ jouent à l'intérieur de la langue $\mathrm{L}_{2}$; le même est vrai des configurations d'unités linguistiques, dans les deux textes.

(27) ne peut pas être considéré comme une définition rigoureuse à cause de l'exigence (ii), qui n'a pas de sens précis: 'jouer un rôle à l'intérieur d'une langue' est assez vague. Mais, si vague qu'elle soit, l'exigence (ii) est très importante, car elle permet de distinguer deux sens du terme traduction. On parlera de traduction $_{1}$, ou de 'traduction faible', quand seule l'exigence (i) est satisfaite; on appellera traduction ${ }_{2}$, ou 'traduction forte', un texte dans la langue d'arrivée qui satisfait aux deux exigences.

Il devient clair que la traduction, (faible) est toujours possible: si on se permet n'importe quels moyens linguistiques, ( $y$ inclus même des moyens META-linguistiques ${ }_{1}$ ), on peut exprimer dans toute langue tout ce qu'on veut.

Par contre, la traduction ${ }_{2}$ (forte) s'avère impossible dans des cas fort nombreux: un sens donné ne peut pas être exprimé dans une langue donnée avec n'importe quel moyen linguistique . $_{\text {. }}$

Là, où seul le sens compte (technologie, science, ...) on peut traduire plus ou moins aisément; mais dans un domaine où les moyens d'expression sont aussi (et parfois même plus) importants que le sens (belles-lettres, et surtout poésie) la traduction peut devenir plus ou moins impossible. Ce résultat doit réconcilier les deux points de vue extrêmes cidessus: les premiers parlent de traduction forte, les deuxièmes, de traduction faible ${ }^{13}$.

Prenons un exemple: le suffixe diminutif -ito/-ita de l'espagnol (qui peut être attaché aux noms et aux adjectifs). D'après $A$. Wierzbicka (sous presse: 15-21), le sens de ce suffixe peut être représenté de la façon suivante:

(28) $X$-ito = 'je pense à $X$ comme à quelque chose de petit et j'ai de bons sentiments envers $\mathrm{X}$ comme on en a normalement envers quelque chose de petit'.

Si cette représentation sémantique de -ito est correcte (et je crois qu'elle l'est) les phrases espagnoles citées sous (29) ne sont pas traduisibles en français (au sens fort):

(29) El niño tiene dos añitos $\approx$ 'L'enfant a deux ans';

Son unos tomatos duritos, colorados $\approx$ 'Ce sont des tomates dures et rouges';

Estaba sentadita en el suelo $\approx$ 'Elle était assise sur le plancher';

El pobre esta muy cansadito $\approx$ 'Le pauvre est très fatigué'.

Dans toutes ces phrases, quand on les traduit en français on perd le sens du (28) - si l'on veut avoir une vraie traduction française, et non une explication scientifique (bien que faite en français) du sens du suffixe -ito. 
Peut-on enfin conclure qu'on a trouvé un pur problème de traduction? L'intraduisibilité doit bien être quelque chose qui ne relève que de la traduction, voyons! Mais, malgré tout, ce n'est pas le cas. Le problème est encore une fois un PROBLÈME D'EXPRESSION. Ou, en d'autres mots: le sens du suffixe -ito une fois compris (pour le comprendre, il n'y a pas de problème), il faut l'exprimer en français ou bien le laisser inexprimé. Et cela, c'est la question globale de la sémantique linguistique ${ }_{2}$ : étudier les sens (ou les classes de sens) qui peuvent être exprimés dans une langue donnée et ceux qui ne le peuvent pas, de même qu'établir les règles de remaniement du sens de départ pour le rendre plus acceptable dans la langue d'arrivée ${ }^{14}$. C'est qu'en réalité, il existe une rétroaction entre le sens et le texte: le texte en cours d'élaboration pour un sens donné exerce une influence importante sur ce sens en le restructurant: le réduisant dans certains endroits et en exigeant le développement dans d'autres. Un Anglais dit I have a cousin; mais le français exige que le sens de cette phrase soit complété par l'indication du sexe, car en français on est obligé de choisir entre cousin et cousine. C'est très intéressant et très pertinent: mais le problème rentre, dans son intégralité, dans la compétence de la linguistique, plus précisément, de la sémantique.

Il est vrai que pour les recherches portant sur l'exprimabilité des sens dans une langue quelconque, la traduction vers cette langue est un instrument excellent: les particularités sémantiques de la langue d'arrivée simplement sautent aux yeux quand on essaie de l'utiliser comme véhicule pour un sens «étranger». Mais en principe, sur le plan théorique, la traduction n'a rien à voir ici, car c'est toujours le problème d'expression (comparez la note 14).

En dehors de (i) - (iv), il n'y a plus, autant que je sache, de soi-disant "problèmes de traduction ". Or, ces quatre problèmes, passés en revue, ne sont pas, comme on vient de voir, des problèmes de traduction non plus. Ce sont des problèmes normaux de toute

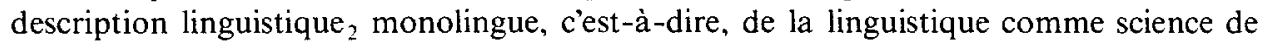
langage.

\section{CONCLUSIONS} courtes.

Après une discussion si longue, les conclusions qu'on peut en tirer peuvent être assez

La science de la traduction, comme science séparée de la linguitique et même, dans un certain degré, opposée à celle-ci, n'existe pas: la linguistique EST la science de la traduction.

Pendant des décennies, on se fatiguait à répéter que les résultats de la linguistique, théorique et comparée, sont vitaux pour la traduction. "The scientific study of translating can and should be regarded as a branch of comparative linguistics... with a focus upon semantics ", - a écrit E. Nida (1975: 95), et cette même idée émerge dans les travaux de tous les spécialistes éminents de la traduction: M. Wandruszka, V. Ju. Rozencvejg, J. Darbelnet et plusieurs autres. (Un exposé excellent de ce point de vue et une bibliographie pertinente peuvent être trouvés dans Nida 1964 et Nida-Taber 1969.)

Cela est sans doute vrai, mais le temps est venu de changer et de mettre l'accent sur le fait inverse: les résultats obtenus dans la théorie et la pratique de la traduction ne sont pas moins vitaux pour la linguistique. En d'autres mots: 


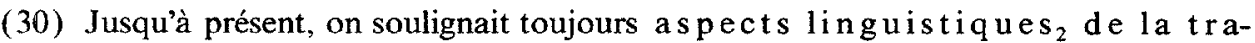
duction (voir par ex., Jakobson 1971b); maintenant, il faut insister davantage sur les aspects «traductionnels»de la linguistique.

Puisque les langues humaines (et LA LANGUE HUMAINE) sont des dispositifs translatifs qui fonctionnent en transformant soit un sens donné dans des textes correspondants, soit un texte donné dans des sens correspondants, la linguistique, science des langues humaines, doit devenir une science de la traduction au sens large du terme. "Comment peut-on exprimer de tels sens dans cette langue?" et "Comment peut-on comprendre de tels textes de cette langue? $"$ - ce sont les deux types de questions principales qui attendent une réponse de la linguistique synchronique, celle-ci pouvant alors se vanter d'être une science strictement en fonction de sa capacité de la donner. Il existe déjà une tradition très prometteuse, enracinée surtout dans la linguistique française: la tradition initiée par des livres tels que Bally 1932 ou Brunot 1936 et continuée de nos jours, par exemple, par Darbelnet 1977, où l'auteur décrit les moyens disponibles en français et en anglais pour l'expression de diverses unités et configurations sémantiques (nombre et quantité, degrés et mesures, l'espace et le mouvement, pouvoir et devoir, détermination - identification - précision, etc.). C'est cette tradition qui constitue, selon mon avis, la voie capitale de la linguistique moderne.

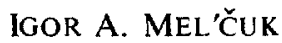

\section{Remerciements}

Ce travail a été écrit à l'invitation de Brian Harris pour une conférence à l'Université d'Ottawa, dans la série des Conférences Vanier.

Une première variante du texte a été lue par L.N. Iordanskaja, dont plusieurs remarques et suggestions ont beaucoup contribué à sa forme finale. Les périphrases (9) et (10) pour l'exemple (8) m'ont été offertes par Guy Connolly. André Clas et Anne-Marie Connolly ont bien voulu corriger le français du manuscrit; Yves-Charles Morin y a donné, en bon ami, les dernières retouches. Enfin, j'ai pu profiter des suggestions de Jean Darbelnet.

C'est une obligation fort plaisante que d'exprimer ici ma gratitude la plus profonde envers tous les amis et collègues mentionnés.

\section{NOTES}

1. "Mais ce sont précisément les banalités que l'on oublie le plus souvent" (Jakobson 1971a: 277).

2. L'adjectif français linguistique est ambigu. Il signifie soit 'relatif au langage et aux langues' (all. sprachlich) soit 'relatif à la linguistique' (all. sprachwissenschaftlich). Comme cette distinction est fondamentale dans le cadre de cet exposé, les deux significations seront différenciées à l'aide des indices: linguistique $_{1}=$ 'relatif au langage ...' linguistique $_{2}=$ 'relatif à la linguistique'.

3. Pour un exposé détaillé de la théorie des MST, voir Mel'cuk 1974a; une esquisse plus courte en peut être trouvée dans Mel'čuk $1973(=1976: 26-62)$ et dans Mel'čuk 1974b. Le travail sur les MST a été commencé, il y a 13 ans, par A.K. Žolkovskij et l'auteur: Žolkovskij-Mel'cuk 1967.

4. Cela veut dire que cette langue sémantique, prise dans son intégralité, n'est pas universelle: chaque 
langue naturelle présuppose sa langue sémantique particulière, toutes ces langues sémantiques ayant, cependant, un NOYAU COMMUN, constitué des sens élémentaires. Les langues sémantiques spécifiques se différencient par la façon d'introduire les abréviations, puisque cette façon est conditionnée par le stock existant de lexèmes de la langue naturelle correspondante.

5. Notez que (7) n'est pas en français normatif, stylistiquement correct : ce qu'on a ici, c'est L'USAGE MÉTALINGUISTIQUE du français, exempt des normes courantes. Les lexèmes. français sont employés dans (6) et (7) seulement à titre d'étiquettes pratiques.

6. Une pareille démarche vis-à-vis des SemR a été entreprise et élaborée dans l'ouvre importante de Th.R. Hofmann et J.-P. Paillet; il s'agit de leur théorie de "réseaux C» $(C$-net theorr $)$. Voir, par exemple, Hofmann 1974 et Paillet 1975.

7. Pour mieux comprendre pourquoi l'introduction des niveaux intermédiaires peut faciliter la description d'une correspondance trap compliquée, il suffit d'examiner un exemple hypothétique : la correspondance entre un objet queiconque $X$ et 100 autres objets $1,2, \ldots, 99,100$.

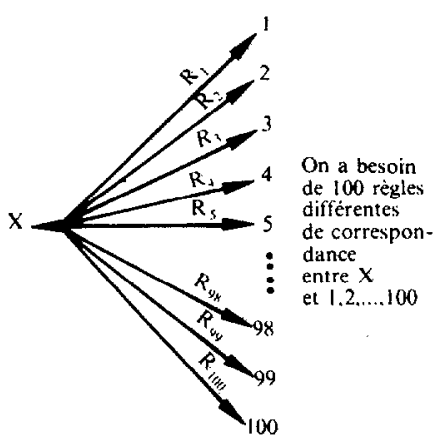

Sans niveaux intermédiaires (correspondance directe)

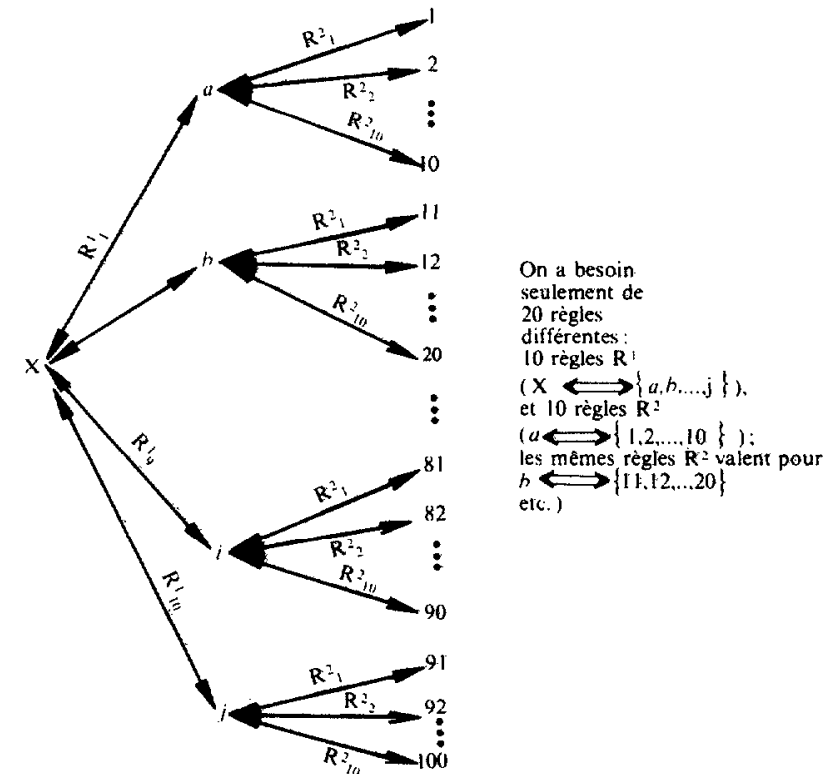

Avec un niveau intermédiaire (objets intermédiaires $a, b, \ldots, j)$

Il est clair que l'économie indiquée n'est peut-être atteinte qu’à la condition suivante: les faits à décrire doivent permettre l'utilisation des mêmes règles entre toutes les représentations du niveau intermédiaire et les représentations plus proches de la surface; dans notre cas, les règles qui font correspondre à l'objet intermédiaire $a$ les objets de surface $1,2, \ldots, 10$ doivent être identiques aux règles qui font correspondre à l'objet intermédiaire $b$ les objets de surface $11,12, \ldots, 20$, et ainsi de suite. On est heureux de trouver une situation semblable dans les langues naturelles.

8. Évidemment, seules les prosodies liées d'une façon directe au sens doivent être indiquées dans (15) et (16); aucune mention n'est faite de prosodies que la syntaxe détermine. - L'analyse sémantique ou syntaxique des phrases écrites sans prosodies marquées est fort répandue, bien que ce soit la même erreur que d'analyser la forme orthographique des mots au lieu de leur composition phonémique. On dit, par exemple, souvent qu'une phrase russe comme

(i) Vse ne spali, lit. 'Tous ne dormaient pas' est ambiguë entre

(ii) 'Il est faux que tous dormaient' [au moins quelques-uns ne dormaient pas, mais d'autres pouvaient bien dormir]

et

(iii) 'Personne ne dormait'.

Il est vrai que la phrase (i) peut être lue à haute voix de deux façons différentes, correspondant aux sens (ii) et (iii), mais avec une prosodie déterminée, elle n'a toujours qu'un seul sens: 
(iv) Vsell ne spali signifie seulement (ii)

9. Pour expliquer pourquoi la proposition relative qui ai dit de (16) est analysée, dans la SSyntS de (19), comme dépendante de $c e$ et non de moi ( on a donc 'ce qui al dit ... est moi' au lieu de 'c'est moi qui ai dit') il suffit de citer la phrase suivante de Mérimée:

(i) C'est mon père, qui a l'habitude des armes, qui a dit cela.

On aperçoit tout de suite la différence essentielle entre la première relative, qui est descriptive, ou qualificative, de l'expression mon père ('mon père, lui, il a l'habitude des armes'), et la deuxième, qui ne sert que pour une mise en relief logique de la même expression ('celui qui a dit ça est mon père, et pas quelqu'un d'autre').

On peut même construire une paire minimale: la phrase

$$
\text { C'est un garçon qui vient d'arriver }
$$

répond à la question 'Qui est-ce?', tandis que la phrase

(iii) C'est un garçon / qui vient d'arriver

répond à la question 'Qui vient d'arriver?' Puisque ces deux phrases ne se différencient que par des moyens syntaxiques (à savoir, par des prosodies syntaxiques) et ont des représentations sémantiques différentes leurs SSyntS doivent être également différentes. On peut relier la relative toujours à garcon: il faut alors introduire une nouvelle relation syntaxique de surface (distincte des SSynt Rel "restrictive " et "descriptive "). Ou bien on peut garder le répertoire des SSyntRel intact en établissant le lien syntaxique entre la relative et ce (au lieu de garçon) et en le traitant comme un cas de SSyntRel «restrictive ". On a choisi la deuxième solution.

10. En réalité, les règles de placement pour les clitiques français sont beaucoup plus compliquées: voir Iordanskaja 1978. - Notons seulement en passant que les expressions incorrectes du type *II yous leur recommandera ou * Je te lui donne (au lieu de $I /$ vous recommandera à eux et Je te donne à lui) sont exclues par des règles-filtres: conditions imposées sur la structure syntaxique de surface qui peuvent faire appel à d'autres niveaux.

11. Nida compare le traducteur à un randonneur qui a à traverser une rivière:

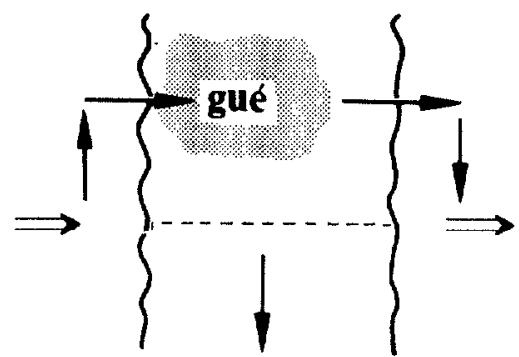

comme il ne peut pas la traverser dans un endroit trop profond (pointillé) il est obligé de remonter le courant, trouver un gué, traverser - et alors redescendre sur son chemin (flèche double). "Le gué", c'est le sens. Voilà ce que Nida écrit: "The translator first analyzes the message of the source language into its simplest and structurally clearest forms, transfers it at this level $\left[=\right.$ le gué $\left.!^{\prime}-1 . M.\right]$, and then restructures it to the level in the receptor language which is most appropriate for the audience he intends to reach » $(1975: 79-80)$.

12. On a ici affaire à ce que nous appelons des fonctions le x icales. Une fonction lexicale est un sens assez abstrait ou un rôle syntaxique profond qui est exprimé par des unités lexicales en fonction d'autres unités lexicales. On peut dire, autrement, que l'expression, ou la vale u r, d'une fonction lexicale est toujours phraséologiquement liée par le lexème qui en est la rgument. 
Voici trois exemples.

- Pour exprimer le sens de 'subir quelque chose', on dit en français:

pour

$\begin{array}{llll}\text { pour } & & \\ \text { approbation } & \text { - recevoir, } & \text { oubli } & \text { - tomber dans, } \\ \text { refus } & \text {-essuger, } & \text { admiration } & \text { - soulever, exciter, } \\ \text { persécutions } & - \text { subir, être } & \text { tourments } & \text {-éprouver. } \\ & \text { en butte à. } & \text { risque, danger } & \text { - courir, ... }\end{array}$

(fonction lexicale Oper $_{2}$ ).

- Pour exprimer le sens 'le son typique de...' on dit en français:

pour

les feuilles mortes - craquer.

un bracelet - tinter.

le sable $\quad$ - crisser, crier.

desferrailles - cliqueter.

applaudissement - crépiter,

un lion

- rugir,

- mugir

$\begin{array}{ll}\text { un ane } & \text { - braire, } \\ \text { un grillon } & \text { - grésiller, }\end{array}$

le tonnerre - gronder...

(fonction lexicale Son).

- Pour exprimer le sens 'très', 'dans un degré élevé', on dit en français:

pour

une lacune

un soutien

un sout
geler

contraintes

critique

- flagrante,

- sans borne.

- à pierre fendre.

- puissantes

- virulente,

$\begin{array}{ll}\text { approbation } & \text { - unanime, } \\ \text { satisfaction } & \text {-forte. } \\ \text { danger } & \text { - grand, } \\ \text { surveiller } & \text { - étroitement, } \\ \text { vent } & \text { - viotent, fort, .. }\end{array}$

(fonction lexicale Magn).

Puisqu'on dispose d'une liste finie de fonctions lexicales (une cinquantaine) valables pour toutes les langues du monde, ces fonctions apparaissent comme un appareil puissant de la description linguistique, des combinaisons lexicales phraséologiquement restraintes. Elles sont bienvenues surtout dans des dictionnaires, où on peut remplir pour chaque lexème une formule standard, par exemple:

\begin{tabular}{l|l|l|l}
\hline \multicolumn{1}{|c|}{ fonctions $_{\text {lexèmes }}$} & Oper $_{1}$ & Oper $_{2}$ & Magn \\
\hline $\begin{array}{l}\text { approbation } \\
\text { danger } \\
\text { admiration }\end{array}$ & $\begin{array}{l}\text { donner } \\
\text { présenter } \\
\text { éprouver }\end{array}$ & $\begin{array}{l}\text { recevoir } \\
\text { courir } \\
\text { soulever, } \\
\text { exciter }\end{array}$ & $\begin{array}{l}\text { unanime } \\
\text { grand } \\
\text { forte }\end{array}$ \\
\hline
\end{tabular}

13. A. Wierzbicka, en insistant sur la traduisibilité absolue, pense toujours à la traduction faible (elle le déclare ouvertement dans son ouvrage). Il est évident qu'on ne peut pas considérer comme traduction une expression comme la suivante:

(i) [X's cousin = ] someone who is related to $\mathrm{X}$ because his father and mother can be thought of as a brother and sister of X's father and mother

(Wierzbicka (sous presse): 12). Dans un mot-à-mot de n'importe quelle langue, (i) sera estimé comme une explication scientifique plutôt qu'une expression normale de la langue en question. - Une analyse profonde et révélatrice des problèmes de traduisibilité est offerte par Keenan 1977, où il est démontré d'une façon convaincante que même dans le domaine de sens une traduisibilité n'est pas toujours possible (entre autres, à cause des présuppositions différentes, etc.).

14. La transformation de l'input sémantique en vue de l'adapter mieux aux exigences de la langue d'arrivée est une opération absolument nécessaire dans le cadre du comportement linguistique, humain Chaque langue impose ses impératifs et ses préférences, non pas seulement sur les textes (ce qui est tout à fait banal), mais aussi sur les sens (ce qui est moins banal). Quant on a affaire à une rue à sens unique, le français dit Accès / Sens interdit, tandis que l'anglais préfère Do not enter. Les sens sont bien différents quoiqu'ils déterminent une même situation réelle. Et pourtant, les DEUX sens sont très facilement exprimables dans les deux langues: N'entrez pas ou Ne vous engagez pas et Access forbidden. Néanmoins, chacune d'elles insiste sur son sens préféré, ce qui s'explique d'ailleurs par des raison linguistiques, (voir cet exemple et son analyse dans Darbelnet 1971:19).

On retrouve des phénomènes similaires à celui-ci un peu partout, et non pas seulement dans le domaine đu langage officiel. - Otojdi ot okna! $\approx$ lit. 'Retire / Écarte-toi de la fenêtre!' dit un Russe à sa petite fille pour qu'elle ne prenne pas froid (on est en hiver, et il y a des courants d'air près de la fenêtre). Un 
Français devrait dire, pour exprimer le même concept, - Ne reste pas près de la fenêtre! (On peut trouver une analyse instructive de ce genre de divergences sémantiques entre le russe et le français dans Rozencvejg 1974.) Lorsque l'anglais dit plutôt high and low, sooner or later, more and less, la langue hindi préfère našib of araz 'low and high', der sower $\approx$ 'later or sooner', $k$ '̀m o bes 'less and more'. C'est-à-dire, que l'ordre des sens correspondants est interverti (Cooper and Ross 1975, où on s'occupe de régularités qui régissent l'ordonnance des sens conjoints).

Bref, sans multiplier nos exemples, il faut insister davantage sur le fait que pour chaque description linguistique ${ }_{2}$ assez complète et pénétrante d'une langue, il faut prévoir un composant responsable pour la formation des représentations sémantiques qui satisfassent aux normes de la langue donnée. Ce problème posé d'une façon explicite (autant que je sache) dans Rozencvejg 1974 ouvre un domaine de recherches complètement nouveau: explorer les lois formelles D'ORGANISATION DES SENS c'est-à-dire, d'organisation du côté sémantique des langues naturelles, Il est vrai que les particularités sémantiques d'une langue sont découvertes plus facilement quand on traduit à partir de cette langue ou vers cette langue. Mais, en principe, comme j’ai déjà eu plusieurs occasions de le dire, les recherches pertinentes ne relèvent pas de la traduction comme telle. Elles sont nécessaires et possibles tout en restant à lintérieur de la langue étudiée.

\section{BIBLIOGRAPHIE}

BALLY, Charles, Linguistique générale et linguistique française. Paris, 1932.

BELINGA SINDEU, Bilounga, "La traduction de la Bible en boulou », Meta, 1977, vol. 22, $n^{\circ}$ 3, $192-194$.

BRUNOT, Ferdinand, La Pensée et la langue; méthode, principes et plan d'une théorie nouvelle du langage appliquée au français, Paris, Masson, 1936.

COOPER, William et John R. ROSS, «World order», In: Grossman, R.E., San, L.J., Vance, T.J. (eds), Papers from the parasession on functionalism, Chicago, Chicago Linguistic Society, 1975, 63-111.

DARBELNET, Jean, “Linguistique différentielle et traduction », Meta, 1971, vol. 16, nos 1-2, 17-24

DARBELNET, Jean. Pensée et struciure, New York, Scribner \& Sons, 1977.

DELISLE, Jean, “Comment traduire "to affect»?», Meta, 1976, vol. 21, n4, 274-277.

EoLS = Essay's on Lexical Semantics, vol. I-Il, ed. by V. Ju. Rozencvejg, Stockholm, Skriptor, 1974.

GAK, Vladimir G., Sopostavitel'naja leksikologija [Lexicologie constrastive], Moscou, Meždun. otnošenija, 1977.

HOFMANN. Thomas R., "In support of semantic atoms", Language sciences, 1974, issue 32, $12-18$.

IORDANSKAJA, Lidija, «Placement des clitiques pronominaux non-sujets dans la phrase française", 1978 (manuscrit).

JAKOBSON, Roman, "Zeichen und System der Sprache", In: R. Jakobson, Selected writings, vol. II, 1971, The Hague, Mouton \& Cie, 272-279 (a).

JAKOBSON, Roman, "On linguistic aspects of translation », idem, 260-266(b).

KEENAN, Edward L.., "Some logical problems in translation ", in F. Guenthner et M. Reutter-Guenthner (eds), Meaning and Translation: Philosophical and Linguistic Approaches, London, Gerald Duckworth and Co., 1977.

MALBLANC, Alfred, Stylistique comparée du fransais et de lallemand, Paris, Didier, 1961.

MEL'ČUK, Igor, "Towards a linguistic "Meaning $\longleftrightarrow$ Text" model", In: F. Kiefer (ed.), Trends in Soviet theoretical linguistics, Dordrecht, Reidel, 1973, 33-57.

MEL'ČUK, Igor, Opyt teorii lingvističeskix modelej «Smysl $\longleftrightarrow$ Tekst ", Moscou, Nauka, 1974(a) [ Essai d'une théorie des modèles linguistiques du type "Sens $\longleftrightarrow$ Texte»].

MEL'ČUK, Igor, «Esquisse d'un modèle linguistique du type "Sens $\longleftrightarrow$ Texte", In : Problèmes actuels en psycholinguistique. Colloques internationaux du CNRS, $\mathrm{n}^{\circ} 206$, Paris, 1974, 291-317(b).

MEL' ̌́U.K, Igor, Das Wort, München. W. Fink Verlag, 1976.

MEL'ČUK, Igor et Aleksandr ŽOLKOVSKIJ, «Towards a functioning Meaning - Text model of language $\%$, Linguistics, 1970, n⿳5口, 10-47.

MPiPL $1964=$ Mašinnyj Perevod i Prikladnaja Lingvistika, vol. 8, 1964 [Traduction automatique et linguistique appliquée ], Trudy I-go Mosk. Gosud. Pedagog. Instituta Inostr. Jazykov. (La plupart des articles contenus dans MPiPL 1964 se trouvent maintenant traduits en anglais en EoLS.)

NAKOS-AUPETIT, Dorothy, “La recherche ponctuelle en terminologie comparée ", Meta, 1977, vol. 22, n०4, 258-263. 
NIDA, Eugene, Toward a science of translating, Leiden, Brill, 1964.

NIDA, Eugene, Language structure and translation, Stanford, Stanford Univ. Press, 1975.

NIDA, Eugene et Charles TABER, The theory and practice of translation, Leiden, Brill, 1969.

PAILlET, J.-P., « Problèmes de notation pour l'étude du contenu linguistique ", Langage, 1975, n 32, 27 69.

ROZENCVEJG, Victor, Opyt lingvističeskogo opisanija leksiko-semantičeskix ošibok y reči na nerodnom jazlke [Essai d'une description linguistique des erreurs lexico-sémantiques de sujets parlant une langue étrangère ], Moscou, 1974. (Institut Russkogo jazyka AN SSSR. Problemnaja gruppa po èksperim, i prikladnoj lingvistike. Predvaritel'nye publikacii, Vypusk 50).

WIERZBICKA, Anna, Semantic primitives, Frankfurt/M, Athenaum, 1972.

WIERZBICKA, Anna. Translatability and semantic primitives (in print).

ŽOLKOVSKIJ, Aleksandr, Quatre articles en EoLS 1974, 155-234.

ŽOLKOVSKIJ, Aleksandr et Igor MEL'ČUK, "O semantičeskom sinteze ", Problem. kihernetiki, 1967, $\mathrm{n}^{\circ}$ 19. 177-238. (Voir la traduction française de cet article: "Sur la synthèse sémantique», T.A. informations, $1970, \mathrm{n}^{\circ} 2$.)

Addenda:

Jaimerais mentionner, à propos des idées exposées ci-dessus, un article de B. Harris qui, malheureusement, m'est tombé sous la main après lachèvement complet du présent ouvrage: «La traductologie, la traduction naturelle, la traduction automatique et la sémantique ", Cahier de linguistique, $\mathrm{n}^{\circ} 2,1973,133-146$. J'ai trouvé dans le dit article beaucoup de thèses que je partage et une proximité de nos points de vue qui me réjouit. 